\title{
Cooperative Spectrum Sensing Techniques with Temporal Dispersive Reporting Channels
}

\author{
Angela Sara Cacciapuoti, Marcello Caleffi, Domenico Izzo, and Luigi Paura
}

\begin{abstract}
Cooperative approaches have been proposed as an effective way to improve the spectrum sensing accuracy. Generally, cooperative spectrum sensing techniques require two successive stages: sensing and reporting. The reporting channels are usually assumed ideal. In this paper, we remove this assumption and we investigate the effects of reporting channels affected by temporal dispersion on cooperative spectrum sensing. To this aim, we propose two fusion schemes: a Widely Linear scheme and a Linear one. For both the schemes, closed-form expressions of the detection and the false alarm probabilities are derived. The performance are also evaluated numerically, and the results show that the Widely Linear detector outperforms the Linear one in operative conditions of practical interest. Moreover, for the sake of completeness, a theoretical comparison of the proposed detectors is carried out for reporting channels affected by multipath frequency non-selective fading. Surprisingly, the analysis proves that the two detectors perform exactly the same under this assumption. Therefore, there is not anymore advantage in using the Widely Linear scheme, which exhibits higher, although limited, computational complexity. The theoretical analysis is validated numerically.
\end{abstract}

Index Terms-Spectrum Sensing, cognitive radio, cooperative spectrum sensing, time dispersive channel.

\section{INTRODUCTION}

$\mathbf{T}$ HE Cognitive Radio (CR) paradigm [1] proposes to enhance the spectrum utilization by allowing unlicensed users (CR users) to utilize dynamically and opportunistically the spectrum assigned to the primary users (PUs), when it is temporarily not used. This opportunistic use of the licensed spectrum has to occur in a controlled fashion through an effective spectrum sensing able to avoid harmful interference against the PUs. The wireless channel hostilities, such as multipath fading and/or shadowing, can compromise the sensing reliability, and hence the PU transmissions. It is possible to improve significantly the sensing accuracy through cooperative approaches [1]-[4], which exploit the inherent spatial diversity among the CR users.

Cooperative detection can be implemented by using a fusion

Manuscript received December 4, 2010; revised April 16, 2011; accepted July 15,2011 . The associate editor coordinating the review of this paper and approving it for publication was X. Wang.

A. S. Cacciapuoti, M. Caleffi, and L. Paura are with the Dept. of Biomedical, Electronics and Telecommunications Engineering, University of Naples Federico II, Naples, Italy (e-mail: \{angelasara.cacciapuoti, marcello.caleffi, paura\}@unina.it).

D. Izzo and L. Paura are with the Laboratorio Nazionale di Comunicazioni Multimediali (CNIT), Naples, Italy (e-mail: \{domenico.izzo\}@cnit.it).

This work is partially supported by the projects "HarBour traffIc opTimizAtion sysTem (HABITAT)" and "DRIVEr monitoring: technologies, methodologies, and IN-vehicle INnovative systems for a safe and ecocompatible driving (DRIVE $\mathrm{IN}^{2}$ )" founded by the Italian national program Piano Operativo Nazionale Ricerca e Competitivit 2007-2013.

Digital Object Identifier 10.1109/TWC.2011.081011.102164 center (FC) that collects and combines the local sensing statistics obtained by each cooperative CR user, for taking the decision on the presence of the PU [3]-[8]. Usually, cooperative sensing requires two successive stages: sensing and reporting [9]. The sensing channels (SCs) between the $\mathrm{PU}$ and the CR users are generally assumed as Rayleigh fading channels with additive white Gaussian noise (AWGN), whereas the reporting channels (RCs), modeling the links between the CR users and the FC, are usually assumed error-free or affected by AWGN [2], [5]-[8], [10]. In practice, however, multipath fading and/or shadowing affect the RCs, causing significant cooperative performance degradation. Therefore, recently, more realistic RC models have been considered.

In [11], the authors analyze the performance of cooperative sensing by adopting an amplify-and-forward relay protocol, when both the SCs and RCs are subjected to correlated shadow-fading. In [12], the authors investigate the impact of correlated shadowing in both the SCs and RCs on cooperative sensing, by showing that the correlated shadowing significantly degrades the sensing performance. In [9], the authors consider both the SCs and RCs modeled as Rayleigh channels, showing that cooperative sensing with many CR users could increase the detection probability only within a limited range. Moreover, the authors propose a sensing technique, which exploits both the space-time and space-frequency coding for counteracting the fading effects. In [13], the authors carry out an outage probability analysis of cognitive transmissions over Rayleigh channels, showing numerically that a minimized outage probability can be achieved through a tradeoff between the sensing time and the data transmission one.

Although all the mentioned works consider feasible RC models, to the best of our knowledge the temporal dispersive model has not yet been adopted for the RCs. Such a model accounts for the RC bandwidth scarcity [14] that can be due to several factors, such as the presence of a large number of cooperative CR users.

Motivated by the previous considerations, in this paper we adopt a linear multipath frequency-selective channel to model each RC between a cooperative CR user and the FC. By accounting for this channel model, we propose a cooperative sensing technique able to exploit the multipath nature of the RCs through a widely-linear (WL) processing, which jointly elaborates the received signal and its conjugate version. The WL fusion rule is designed by maximizing at the FC side the deflection coefficient [15], modified to account for the cognitive radio context. The modified deflection coefficient has been proposed in [6] for designing a linear (L) combining $\mathrm{FC}$ rule in a scenario characterized by $\mathrm{RCs}$ affected by only AWGN. In this work, we assume as local CR sensing 
technique the widely adopted energy detector [2], [5], [6], [8], [9], [12], since it is the simplest one and it does not require any a-priori knowledge on the PU. Moreover, for the sake of comparison, we develop a $\mathrm{L}$ fusion rule, by adopting the same strategy used for the WL fusion rule, i.e., the maximization of the modified deflection coefficient.

For both the proposed techniques, we provide closed-form expressions of the detection and false-alarm probabilities. Numerical results show that the WL detector outperforms the $\mathrm{L}$ one in operative conditions of practical interest, assuring a more reliable detection of the PU at the price of a small complexity increasing. The WL performance gain is justified by its ability to take advantage of the non-circular (or improper) properties [16], [17] of the local sensing statistics, since it exploits the information contained in the statistical pseudocovariance function of the received signal.

Finally, for the sake of completeness, we carry out a theoretical analysis to compare the proposed techniques when the RCs are affected by multipath frequency non-selective fading, i.e., when there is not temporal dispersion. Surprisingly, the analysis proves that the two strategies perform exactly the same under this assumption. Therefore the WL scheme, which exhibits higher although limited computational complexity, is not anymore advantageous with respect to the $\mathrm{L}$ one. The theoretical analysis is then validated numerically.

In a nutshell, the contribution of this paper is twofold: i) the design of two cooperative techniques that take into account the temporal dispersive nature of the RCs; ii) the theoretical comparison of the proposed detectors when the $\mathrm{RCs}$ are affected by multipath frequency non-selective fading.

The paper is organized as follows: Section II describes the system model. In Section III, the WL and L techniques are designed. The theoretical comparison of the proposed techniques is given in Section IV. Section V provides simulation results. Finally, conclusions are drawn in Section VI, and some proofs are gathered in Appendix A and B.

\section{SySTEM MOdeL}

In this Section, we describe first the system model at each CR user, then the model at the FC, by accounting for temporal dispersive RCs. ${ }^{1}$

\section{A. The CR user Model}

We consider a CR network with $M$ cooperative CR users. The base-band discrete received signal $x_{i}(k)$ at the $i$-th $\mathrm{CR}$ user can be written as:

$$
x_{i}(k)=\left\{\begin{array}{ll}
v_{i}(k) & \mathcal{H}_{0} \\
g_{i} s(k)+v_{i}(k) & \mathcal{H}_{1}
\end{array} \quad \forall i \in\{1, \ldots, M\}\right.
$$

${ }^{1}$ Notations: $\mathcal{H}_{0}$ and $\mathcal{H}_{1}$ denote the hypotheses of absence and presence of the PU, respectively; the fields of complex and real numbers are denoted with $\mathbb{C}$ and $\mathbb{R} ;$ matrices [vectors] are denoted with upper case [lower case] boldface letters (e.g., $\mathbf{X}$ or $\mathbf{x}$ ); the field of $m \times n$ complex [real] matrices is denoted as $\mathbb{C}^{m \times n}\left[\mathbb{R}^{m \times n}\right]$, with $\mathbb{C}^{m}\left[\mathbb{R}^{m}\right]$ used as a shorthand for $\mathbb{C}^{m \times 1}\left[\mathbb{R}^{m \times 1}\right]$; the superscripts $*, T, H$, and -1 denote the conjugate, the transpose, the Hermitian (conjugate transpose) and the inverse of a matrix, respectively; $\mathbf{I}_{m} \in \mathbb{R}^{m \times m}$ denotes the identity matrix; for any $\mathbf{a} \in \mathbb{C}^{m},\|\mathbf{a}\|_{2}^{2} \triangleq \sqrt{\mathbf{a}^{H} \mathbf{a}}$ denotes the Euclidean norm; $\mathbf{A}=\operatorname{diag}\left(\mathbf{A}_{11} \mathbf{A}_{22} \ldots \mathbf{A}_{n n}\right)$ is a diagonal matrix with elements $\mathbf{A}_{i i}$ on the main diagonal; E[.] and $\operatorname{Var}[\cdot]$ denote the statistical mean and variance, respectively; $\operatorname{Re}[\cdot]$ denotes the real part. where $s(k)$ is the PU's signal, $g_{i}$ is the complex channel coefficient that models the SC between the PU and the $i$-th CR user. $v_{i}(k)$ is the complex zero-mean AWGN, $v_{i}(k) \sim \mathcal{C N}\left(0, \sigma_{i}^{2}\right)$, assumed circular (or proper) [16], i.e. $E\left[v_{i}(n) v_{i}(m)\right]=0, \forall n, m \in Z$. We assume that each CR user adopts an energy detector as local sensing technique, i.e., $u_{i}(q)=\sum_{k=q N_{s}}^{q N_{s}+N-1}\left|x_{i}(k)\right|^{2}, i \in\{1, \ldots, M\}$, where $N=\left\lfloor\tau f_{s}\right\rfloor[18]$ is the number of samples available in the sensing time, with $\tau$ the available sensing time and $f_{s}$ the sampling frequency. $N_{s}>N$ denotes the number of samples after which a new spectrum sensing process starts. Accounting for the Central Limit Theorem (CLT), for large $N, u_{i}(q)$ is approximated by a Gaussian random variable (r.v.) under both the hypotheses $\mathcal{H}_{0}$ and $\mathcal{H}_{1}$ [18], with mean and variance given by, respectively:

$$
\begin{aligned}
E\left[u_{i}(q) \mid \mathcal{H}_{j}\right]= \begin{cases}N \sigma_{i}^{2} & \mathcal{H}_{0} \\
\left|g_{i}\right|^{2} E_{s}+N \sigma_{i}^{2} & \mathcal{H}_{1}\end{cases} \\
\operatorname{Var}\left[u_{i}(q) \mid \mathcal{H}_{j}\right]= \begin{cases}N \sigma_{i}^{4} & \mathcal{H}_{0} \\
2\left|g_{i}\right|^{2} E_{s} \sigma_{i}^{2}+N \sigma_{i}^{4} & \mathcal{H}_{1}\end{cases}
\end{aligned}
$$

$E_{s}=\sum_{k=0}^{N-1}|s(k)|^{2}$ denotes the PU signal energy over $N$ samples, and $s(k)$ is assumed deterministic and unknown [5][8]. We note that also if $s(k)$ is modeled as a sequence of independent and identically distributed random variables (r.vv.), $u_{i}(q)$ can be still approximated by a Gaussian r.v. [18], and the subsequent analysis continues to hold.

\section{B. The Fusion Center Model}

The statistics $\left\{u_{i}(q)\right\}$ are transmitted to the FC through temporal dispersive RCs (Fig. 1). ${ }^{2}$ Like in [2], [5]-[10], [12], [18], we assume that the transmissions of different CR users are mutually orthogonal ${ }^{3}$ and that the status of the PU remains unchanged during the detection process. The base-band signal at the RF front-end of the FC received from the $i$-th CR user is:

$$
y_{i}(l)=\sum_{n=0}^{L_{g_{i}}-1} u_{i}(l-n) h_{i}(n)+n_{i}(l), \quad i \in\{1, \ldots, M\}
$$

where $l$ is the arbitrary sampling instant at the $\mathrm{FC}$ side, $n_{i}(l) \sim \mathcal{C N}\left(0, \delta_{i}^{2}\right)$ is the circular, complex zero-mean, spatially uncorrelated, AWGN at the FC, and $\left\{h_{i}(n)\right\}_{n=0}^{L_{g_{i}}}$ is the finite multipath channel impulse response with length $L_{g_{i}}$ that models the RC between the $i$-th CR user and the FC. It is reasonable to assume that geographically distributed $\mathrm{CR}$ users experience independent fading. Therefore, $u_{i}\left(l_{1}\right)$ and $u_{j}\left(l_{2}\right)$, $i \neq j$, are conditionally spatially uncorrelated under each hypothesis. Moreover, the transmitted statistics $\left\{u_{i}(l)\right\}$ are assumed independent of the noise $\left\{n_{i}(l)\right\}$. In the following, to simplify the notation, $L_{g}$ denotes the largest channel length,

\footnotetext{
${ }^{2}$ We note that there is no information exchanged among the cooperative $\mathrm{CR}$ users, since they communicate only with the FC that handles the coordination among them. The FC takes also the final decision on the presence of the PU, by combining the local sensing information sent by each CR user.

${ }^{3}$ This assumption, extensively adopted in the literature, and verified if the co-channel interference among the RCs can be neglected, is reasonable satisfied in the considered scenario since the FC cares of the coordination among the CR users.
} 


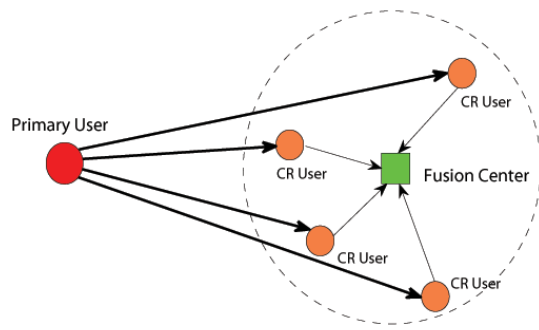

Fig. 1. CR Network with a FC and multiple cooperative CR users.

i.e., $L_{g}=\max _{i} L_{g_{i}}$. At the FC, due to the multipath channel model, we jointly elaborate $L_{e}$ consecutive received samples for each CR user, i.e., $\mathbf{y}_{i}(l) \triangleq\left[y_{i}(l), y_{i}(l-1), \ldots y_{i}\left(l-L_{e}+\right.\right.$ $1)]^{T} \in \mathbb{C}^{L_{e}}$. By using (3), $\mathbf{y}_{i}(l)$ can be expressed as:

$$
\mathbf{y}_{i}(l)=\mathbf{H}_{i} \mathbf{u}_{i}(l)+\mathbf{n}_{i}(l), \quad i \in\{1, \ldots, M\}
$$

where $\mathbf{H}_{i} \in \mathbb{C}^{L_{e} \times\left(L_{e}+L_{g}-1\right)}$ is the Toeplitz upper triangular matrix [19] with the first row $\left[h_{i}(0) \ldots h_{i}\left(L_{g}-1\right) 0 \ldots 0\right]$ and first column $\left[h_{i}(0) 0 \ldots 0\right]^{T} . \mathbf{u}_{i}(l) \triangleq\left[u_{i}(l), u_{i}(l-\right.$ $\left.1), \ldots, u_{i}\left(l-L_{e}-L_{g}+2\right)\right]^{T} \in \mathbb{R}^{L_{e}+L_{g}-1}$ is the real vector of the transmitted energies, and $\mathbf{n}_{i}(l) \triangleq\left[n_{i}(l), n_{i}(l-\right.$ $\left.1), \ldots, n_{i}\left(l-L_{e}+1\right)\right]^{T} \in \mathbb{C}^{L_{e}}$ is the noise vector. Unlike the r.vv. $\left\{u_{i}(m)\right\}_{m=l}^{l-L_{e}-L_{g}+2}$, the r.vv. $\left\{y_{i}(m)\right\}_{m=l}^{l-L_{e}+1}$ are not conditional uncorrelated due to the temporal dispersive RCs. By defining $\mathbf{y}(l) \triangleq\left[\mathbf{y}_{1}^{T}(l) \mathbf{y}_{2}^{T}(l) \ldots \mathbf{y}_{M}^{T}(l)\right]^{T} \in \mathbb{C}^{M L_{e}}$, $\mathbf{H} \triangleq \operatorname{diag}\left[\mathbf{H}_{1} \mathbf{H}_{2} \ldots \mathbf{H}_{M}\right] \in \mathbb{C}^{M L_{e} \times M\left(L_{e}+L_{g}-1\right)}, \mathbf{u}(l) \triangleq$ $\left[\mathbf{u}_{1}^{T}(l) \mathbf{u}_{2}^{T}(l) \ldots \mathbf{u}_{M}^{T}(l)\right]^{T} \in \mathbb{R}^{M\left(L_{e}+L_{g}-1\right)}$, and $\mathbf{n}(l) \triangleq$ $\left[\mathbf{n}_{1}^{T}(l) \mathbf{n}_{2}^{T}(l) \ldots \mathbf{n}_{M}^{T}(l)\right]^{T} \in \mathbb{C}^{M L_{e}}$, (4) can be rewritten as:

$$
\mathbf{y}(l)=\mathbf{H} \mathbf{u}(l)+\mathbf{n}(l)
$$

Since $\mathbf{u}(l)$ is a Gaussian random vector via CLT, $\mathbf{y}(l)$ is a Gaussian random vector as well ${ }^{4}$, with mean and covariance matrix derived in the following by using (2) and (5):

$$
\boldsymbol{\mu}_{\mathcal{H}_{j}}=E\left[\mathbf{y}(l) \mid \mathcal{H}_{j}\right]=\mathbf{H} E\left[\mathbf{u}(l) \mid \mathcal{H}_{j}\right]=\mathbf{H} \boldsymbol{\eta}_{\mathcal{H}_{j}} \in \mathbb{C}^{M L_{e}}
$$

where, by indicating with $\mathbf{1} \triangleq[11 \ldots 1]^{T} \in \mathbb{R}^{L_{e}+L_{g}-1}$,

$$
\begin{aligned}
& \boldsymbol{\eta}_{\mathcal{H}_{j}} \triangleq E\left[\mathbf{u}(l) \mid \mathcal{H}_{j}\right]= \\
& = \begin{cases}{\left[N \sigma_{1}^{2} \mathbf{1}^{T} \ldots N \sigma_{M}^{2} \mathbf{1}^{T}\right]^{T}} \\
{\left[\left(E_{s}\left|g_{1}\right|^{2}+N \sigma_{1}^{2}\right) \mathbf{1}^{T} \ldots\left(E_{s}\left|g_{M}\right|^{2}+N \sigma_{M}^{2}\right) \mathbf{1}^{T}\right]^{T}} & \mathcal{H}_{1}\end{cases} \\
& \mathbf{C}_{\mathbf{y} \mid \mathcal{H}_{j}} \triangleq E\left[(\mathbf{y}(l)-\boldsymbol{\mu})(\mathbf{y}(l)-\boldsymbol{\mu})^{H} \mid \mathcal{H}_{j}\right] \in \mathbb{C}^{M L_{e} \times M L_{e}}= \\
& \quad=\mathbf{H C}_{\mathbf{u} \mid \mathcal{H}_{j}} \mathbf{H}^{H}+\mathbf{R}_{\mathbf{n}}
\end{aligned}
$$

In (8), $\mathbf{R}_{\mathbf{n}}$ and $\mathbf{C}_{\mathbf{u} \mid \mathcal{H}_{j}}$ denote the noise correlation matrix and the transmitted statistics covariance matrix, respectively. Stemming from the previous assumptions, they are equal to:

$$
\begin{aligned}
\mathbf{R}_{\mathbf{n}} & =\operatorname{diag}\left[\delta_{1}^{2} \mathbf{I}_{\left\{L_{e} \times L_{e}\right\}} \ldots \delta_{M}^{2} \mathbf{I}_{\left\{L_{e} \times L_{e}\right\}}\right] \triangleq \\
& \triangleq \operatorname{diag}\left[\mathbf{R}_{\mathbf{n}_{1}} \ldots \mathbf{R}_{\mathbf{n}_{M}}\right] \in \mathbb{R}^{M L_{e} \times M L_{e}}
\end{aligned}
$$

\footnotetext{
${ }^{4} \mathrm{We}$ assume to know the multipath channel impulse responses that model the RCs between the CR users and the FC. For this, $\mathbf{y}(l)$ can be modeled as a Gaussian random vector.
}

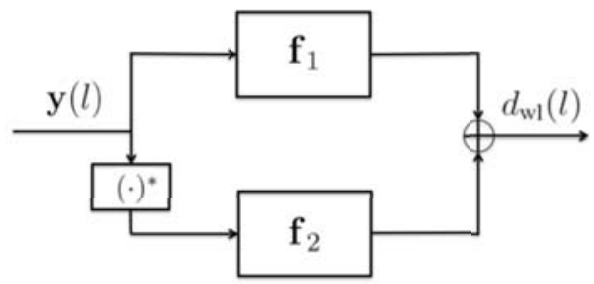

Fig. 2. Widely Linear Processing.

$$
\begin{aligned}
\mathbf{C}_{\mathbf{u} \mid \mathcal{H}_{j}} & \in \mathbb{R}^{M\left(L_{e}+L_{g}-1\right) \times M\left(L_{e}+L_{g}-1\right)}= \\
& =\operatorname{diag}\left[\mathbf{C}_{\mathbf{u}_{1} \mid \mathcal{H}_{j}} \ldots \mathbf{C}_{\mathbf{u}_{M} \mid \mathcal{H}_{j}}\right]
\end{aligned}
$$

with

$$
\begin{aligned}
& \mathbf{C}_{\mathbf{u}_{i} \mid \mathcal{H}_{j}} \triangleq E\left[\left(\mathbf{u}_{i}(l)-\boldsymbol{\eta}\right)\left(\mathbf{u}_{i}(l)-\boldsymbol{\eta}\right)^{H} \mid \mathcal{H}_{j}\right]= \\
& = \begin{cases}N \sigma_{i}^{4} \mathbf{I}_{\left\{L_{e}+L_{g}-1\right\}} & \mathcal{H}_{0} \\
\left(N \sigma_{i}^{4}+2 E_{s}\left|g_{i}\right|^{2} \sigma_{i}^{2}\right) \mathbf{I}_{\left\{L_{e}+L_{g}-1\right\}} & \mathcal{H}_{1}\end{cases}
\end{aligned}
$$

where $\boldsymbol{\eta}_{\mathcal{H}_{j}}$ is given in (7). To derive expression (11), we have exploited the un-correlation and the identical distribution of the r.vv. $\left\{u_{i}(m)\right\}_{m=l}^{l-L_{e}-L_{g}+2}$, and we have taken into account expression (2) of their variance. Plus, the pseudo-covariance matrix of $\mathbf{y}(l)$ is different from zero and it is given by:

$$
\begin{aligned}
\mathbf{C}_{\mathbf{y}^{*} \mid \mathcal{H}_{j}} & \triangleq E\left[(\mathbf{y}(l)-\boldsymbol{\mu})(\mathbf{y}(l)-\boldsymbol{\mu})^{T} \mid \mathcal{H}_{j}\right] \in \mathbb{C}^{M L_{e} \times M L_{e}}= \\
& =\mathbf{H C}_{\mathbf{u} \mid \mathcal{H}_{j}} \mathbf{H}^{T}
\end{aligned}
$$

where the last equality takes into account that $\mathbf{n}(l)$ is a proper Gaussian random vector. Since $\mathbf{C}_{\mathbf{y}^{*} \mid \mathcal{H}_{j}}$ is different by the null matrix, $\mathbf{y}(l)$ exhibits non-circular properties [16].

\section{Cooperative Detection Strategies}

In this section, we design the proposed WL and L cooperative fusion techniques, providing for both the strategies closedform expressions of the false-alarm and detection probabilities.

\section{A. Widely Linear Global Decision}

Since, as shown before, $\mathbf{y}(l)$ exhibits non-circular properties, it exists correlation (12) between the received signal $\mathbf{y}(l)$ and its conjugate version. To exploit the further information contained in its statistical pseudo-covariance function, we propose to consider a WL elaboration that consists in processing not only the received vector, but also its conjugate version, as illustrated in Fig. 2. Hence, a WL global test statistic can be written as:

$$
d_{\mathrm{wl}}(l)=\mathbf{f}_{1}^{H} \mathbf{y}(l)+\mathbf{f}_{2}^{H} \mathbf{y}^{*}(l)
$$

By defining $\mathbf{y}_{a}(l) \triangleq\left[\mathbf{y}^{T}(l) \quad \mathbf{y}^{H}(l)\right]^{T} \in \mathbb{C}^{2 M L_{e}}$, referred to as augmented received vector, and $\mathbf{f} \triangleq\left[\begin{array}{ll}\mathbf{f}_{1}^{T} & \mathbf{f}_{2}^{T}\end{array}\right]^{T}$, referred to as augmented processing vector, it is possible to express (13) as:

$$
d_{\mathrm{wl}}(l)=\mathbf{f}^{H} \mathbf{y}_{a}(l)=2 \operatorname{Re}\left[\mathbf{f}_{1}^{H} \mathbf{y}(l)\right]
$$

where in the last equality we have used the relation $\mathbf{f}_{2}=\mathbf{f}_{1}^{*}$ that holds for real information symbols. Specifically, for real symbols, the augmented processing vector $\mathbf{f}$ exhibits the conjugate symmetric property [20], implying that the statistic (14) is real. Since $\mathbf{y}(l)$ and its conjugate version $\mathbf{y}^{*}(l)$ are 
Gaussian, their WL combination $d_{\mathrm{wl}}(l)$ is also a Gaussian r.v., whose mean and variance is derived by characterizing the random vector $\mathbf{y}_{a}(l)$ in (14). At this end, by using (5), $\mathbf{y}_{a}(l)$ can be expressed as:

$$
\mathbf{y}_{a}(l)=\underbrace{\left[\begin{array}{c}
\mathbf{H} \\
\mathbf{H}^{*}
\end{array}\right]}_{\triangleq \mathbf{H}_{a}} \mathbf{u}(l)+\underbrace{\left[\begin{array}{c}
\mathbf{n}(l) \\
\mathbf{n}^{*}(l)
\end{array}\right]}_{\triangleq \mathbf{n}_{a}(l)}=\mathbf{H}_{a} \mathbf{u}(l)+\mathbf{n}_{a}(l)
$$

and its conditional mean $\widetilde{\boldsymbol{\mu}}_{\mathcal{H}_{j}} \triangleq E\left[\mathbf{y}_{a}(l) \mid \mathcal{H}_{j}\right] \in \mathbb{C}^{2 M L_{e}}$ is equal equivalently to:

$$
\begin{gathered}
\tilde{\boldsymbol{\mu}}_{\mathcal{H}_{j}}=\mathbf{H}_{a} E\left[\mathbf{u}(l) \mid \mathcal{H}_{j}\right]=\mathbf{H}_{a} \boldsymbol{\eta}_{\mathcal{H}_{j}} \\
\tilde{\boldsymbol{\mu}}_{\mathcal{H}_{j}}=\left[\begin{array}{c}
\mathbf{H} \boldsymbol{\eta}_{\mathcal{H}_{j}} \\
\mathbf{H}^{*} \boldsymbol{\eta}_{\mathcal{H}_{j}}
\end{array}\right]=\left[\begin{array}{l}
\boldsymbol{\mu}_{\mathcal{H}_{j}} \\
\boldsymbol{\mu}_{\mathcal{H}_{j}}^{*}
\end{array}\right]
\end{gathered}
$$

with $\boldsymbol{\eta}_{\mathcal{H}_{j}}$ given by (7). (17), by using the definition of $\mathbf{y}_{a}(l)$, expresses $\widetilde{\boldsymbol{\mu}}_{\mathcal{H}_{j}}$ as function of the mean (6) of the unstacked vector $\mathbf{y}(l)$. In addition, the covariance matrix of $\mathbf{y}_{a}(l)$ is

$$
\begin{aligned}
\mathcal{C}_{\mathbf{y}_{a} \mid \mathcal{H}_{j}} & \triangleq E\left[\left(\mathbf{y}_{a}(l)-\tilde{\boldsymbol{\mu}}\right)\left(\mathbf{y}_{a}(l)-\widetilde{\boldsymbol{\mu}}\right)^{H} \mid \mathcal{H}_{j}\right] \in \mathbb{C}^{2 M L_{e} \times 2 M L_{e}} \\
& =\mathbf{H}_{a} \mathbf{C}_{\mathbf{u} \mid \mathcal{H}_{j}} \mathbf{H}_{a}^{H}+\mathbf{R}_{\mathbf{n}_{a}}
\end{aligned}
$$

with $\mathbf{C}_{\mathbf{u} \mid \mathcal{H}_{j}}$ given by (10). In (18), $\mathbf{R}_{\mathbf{n}_{a}} \triangleq \operatorname{diag}\left[\mathbf{R}_{\mathbf{n}}, \mathbf{R}_{\mathbf{n}}\right] \in$ $\mathbb{R}^{2 M L_{e} \times 2 M L_{e}}$ denotes the augmented noise correlation matrix. By using the definition of $\mathbf{y}_{a}(l), \mathcal{C}_{\mathbf{y}_{a} \mid \mathcal{H}_{j}}$ can be also expressed as function of the covariance (8) and pseudo-covariance (12) matrices of the unstacked vector $\mathbf{y}(l)$ :

$$
\mathcal{C}_{\mathbf{y}_{a} \mid \mathcal{H}_{j}}=\left[\begin{array}{cc}
\mathbf{C}_{\mathbf{y} \mid \mathcal{H}_{j}} & \mathbf{C}_{\mathbf{y}^{*} \mid \mathcal{H}_{j}} \\
\mathbf{C}_{\mathbf{y}^{*} \mid \mathcal{H}_{j}}^{*} & \mathbf{C}_{\mathbf{y} \mid \mathcal{H}_{j}}^{*}
\end{array}\right]
$$

By using (16), (18), $d_{\mathrm{wl}}(l)$ in (14) is characterized as follows:

$$
\begin{aligned}
& \mu_{\mathrm{wl} \mid \mathcal{H}_{j}} \triangleq E\left[d_{\mathrm{wl}}(l) \mid \mathcal{H}_{j}\right]=\mathbf{f}^{H} E\left[\mathbf{y}_{a}(l) \mid \mathcal{H}_{j}\right]=\mathbf{f}^{H} \widetilde{\boldsymbol{\mu}}_{\mathcal{H}_{j}} \\
& \varphi_{\mathrm{wl} \mid \mathcal{H}_{j}}^{2} \triangleq \operatorname{Var}\left[d_{\mathrm{wl}}(l) \mid \mathcal{H}_{j}\right]= \\
& =\mathbf{f}^{H} E\left[\left(\mathbf{y}_{a}(l)-\tilde{\boldsymbol{\mu}}\right)\left(\mathbf{y}_{a}(l)-\tilde{\boldsymbol{\mu}}\right)^{H} \mid \mathcal{H}_{j}\right] \mathbf{f}=\mathbf{f}^{H} \mathbf{C}_{\mathbf{y}_{a} \mid \mathcal{H}_{j}} \mathbf{f}
\end{aligned}
$$

Then the FC takes the global decision on the presence of the PU by comparing the WL test statistic $d_{\mathrm{wl}}(l)$ with a decision threshold $\gamma_{\mathrm{wl}}$, i.e.:

$$
d_{\mathrm{wl}}(l) \gtreqless \mathcal{H}_{1} \mathcal{H}_{0} \gamma_{\mathrm{wl}}
$$

Consequently, by accounting for (20) and (21), the false-alarm $P_{f}^{\mathrm{wl}}$ and the detection $P_{d}^{\mathrm{wl}}$ probabilities can be expressed as:

$$
\begin{aligned}
P_{f}^{\mathrm{wl}} & \triangleq P\left[d_{\mathrm{wl}}(l)>\gamma_{\mathrm{wl}} \mid \mathcal{H}_{0}\right]=Q\left(\frac{\gamma_{\mathrm{wl}}-\mu_{\mathrm{wl} \mid \mathcal{H}_{0}}}{\sqrt{\varphi_{\mathrm{wl} \mid \mathcal{H}_{0}}^{2}}}\right)= \\
& =Q\left(\frac{\gamma_{\mathrm{wl}}-\mathbf{f}^{H} \widetilde{\boldsymbol{\mu}}_{\mathcal{H}_{0}}}{\sqrt{\mathbf{f}^{H} \mathbf{C}_{\mathbf{y}_{a} \mid \mathcal{H}_{0}} \mathbf{f}}}\right) \\
P_{d}^{\mathrm{wl}} & \triangleq P\left[d_{\mathrm{wl}}(l)>\gamma_{\mathrm{wl}} \mid \mathcal{H}_{1}\right]=Q\left(\frac{\gamma_{\mathrm{wl}}-\mu_{\mathrm{wl} \mid \mathcal{H}_{1}}}{\sqrt{\varphi_{\mathrm{wl} \mid \mathcal{H}_{1}}^{2}}}\right)= \\
& =Q\left(\frac{\gamma_{\mathrm{wl}}-\mathbf{f}^{H} \widetilde{\boldsymbol{\mu}}_{\mathcal{H}_{1}}}{\sqrt{\mathbf{f}^{H} \mathbf{C}_{\mathbf{y}_{a} \mid \mathcal{H}_{1}} \mathbf{f}}}\right)
\end{aligned}
$$

\section{B. The Widely Linear Optimization}

Here, we single out the augmented processing vector $f$ according to the selected optimization criterion. The statistics (20) and (21) are affected by $\mathbf{f}$ and, consequently, also both the conditional Probability Density Functions (PDFs) of the decision r.v. $d_{\mathrm{wl}}(l)$ depend on $\mathbf{f}$. An effective optimization criterion consists of maximizing the deflection coefficient [15], i.e., the variance-normalized distance between the expectations under the hypotheses $\mathcal{H}_{1}$ and $\mathcal{H}_{0}$. To increase the detection sensitivity for a fixed false-alarm probability, the deflection coefficient is modified [8] by normalizing the distance between the centers of the two conditional PDFs with the variance in the hypothesis $\mathcal{H}_{1}$ :

$$
m_{\mathrm{wl}}^{2}(\mathbf{f}) \triangleq \frac{\left\{E\left[d_{\mathrm{wl}}(l) \mid \mathcal{H}_{1}\right]-E\left[d_{\mathrm{wl}}(l) \mid \mathcal{H}_{0}\right]\right\}^{2}}{\operatorname{Var}\left[d_{\mathrm{wl}}(l) \mid \mathcal{H}_{1}\right]}
$$

By taking into account (20) and (21), (25) can be rewritten as:

$$
m_{\mathrm{wl}}^{2}(\mathbf{f})=\frac{\left[\mu_{\mathrm{wl} \mid \mathcal{H}_{1}}-\mu_{\mathrm{wl} \mid \mathcal{H}_{0}}\right]^{2}}{\mathbf{f}^{H} \mathbf{C}_{\mathbf{y}_{a} \mid \mathcal{H}_{1}} \mathbf{f}}=\frac{\left[\mathbf{f}^{H}\left(\widetilde{\boldsymbol{\mu}}_{\mathcal{H}_{1}}-\widetilde{\boldsymbol{\mu}}_{\mathcal{H}_{0}}\right)\right]^{2}}{\mathbf{f}^{H} \mathbf{C}_{\mathbf{y}_{a} \mid \mathcal{H}_{1}} \mathbf{f}}
$$

To avoid undetermined solutions, (26) is maximized under the unit-norm constraint for $\mathbf{f}$ :

$$
\max _{\mathbf{f}} m_{\mathrm{wl}}^{2}(\mathbf{f}) \quad \text { subject to } \mathbf{f}^{H} \mathbf{f}=1
$$

By using $\left(\mathbf{C}_{\mathbf{y}_{a} \mid \mathcal{H}_{1}}^{1 / 2} \mathbf{f}\right)^{H} \mathbf{C}_{\mathbf{y}_{a} \mid \mathcal{H}_{1}}^{-1 / 2}=\mathbf{f}^{H}$ and the CauchySchwartz's inequality [19], one has:

$$
\begin{aligned}
m_{\mathrm{wl}}^{2}(\mathbf{f}) & =\frac{\left[\left(\mathbf{C}_{\mathbf{y}_{a} \mid \mathcal{H}_{1}}^{1 / 2} \mathbf{f}\right)^{H} \mathbf{C}_{\mathbf{y}_{a} \mid \mathcal{H}_{1}}^{-1 / 2}\left(\widetilde{\boldsymbol{\mu}}_{\mathcal{H}_{1}}-\widetilde{\boldsymbol{\mu}}_{\mathcal{H}_{0}}\right)\right]^{2}}{\mathbf{f}^{H} \mathbf{C}_{\mathbf{y}_{a} \mid \mathcal{H}_{1}} \mathbf{f}} \\
& \leq\left(\widetilde{\boldsymbol{\mu}}_{\mathcal{H}_{1}}-\widetilde{\boldsymbol{\mu}}_{\mathcal{H}_{0}}\right)^{H} \mathbf{C}_{\mathbf{y}_{a} \mid \mathcal{H}_{1}}^{-1}\left(\widetilde{\boldsymbol{\mu}}_{\mathcal{H}_{1}}-\widetilde{\boldsymbol{\mu}}_{\mathcal{H}_{0}}\right)
\end{aligned}
$$

where the equality is achieved if and only if:

$$
\mathbf{f}_{\mathrm{wl}-\mathrm{opt}}=\xi \mathbf{C}_{\mathbf{y}_{a} \mid \mathcal{H}_{1}}^{-1}\left(\widetilde{\boldsymbol{\mu}}_{\mathcal{H}_{1}}-\widetilde{\boldsymbol{\mu}}_{\mathcal{H}_{0}}\right)
$$

The constraint $\mathbf{f}^{H} \mathbf{f}=1$ is satisfied by setting the constant $\xi=1 /\left\|\mathbf{C}_{\mathbf{y}_{a} \mid \mathcal{H}_{1}}^{-1}\left(\widetilde{\boldsymbol{\mu}}_{\mathcal{H}_{1}}-\widetilde{\boldsymbol{\mu}}_{\mathcal{H}_{0}}\right)\right\|_{2}^{2}$, which is a real value to assure the conjugate symmetric property of the augmented vector $\mathbf{f}$. Hence, the modified deflection coefficient is maximized by:

$$
\mathbf{f}_{\mathrm{wl}-\mathrm{opt}} \triangleq\left[\begin{array}{c}
\mathbf{f}_{1, \mathrm{wl}-\mathrm{opt}} \\
\mathbf{f}_{1, \mathrm{wl}-\mathrm{opt}}^{*}
\end{array}\right]=\frac{\mathbf{C}_{\mathbf{y}_{a} \mid \mathcal{H}_{1}}^{-1}\left(\widetilde{\boldsymbol{\mu}}_{\mathcal{H}_{1}}-\widetilde{\boldsymbol{\mu}}_{\mathcal{H}_{0}}\right)}{\left\|\mathbf{C}_{\mathbf{y}_{a} \mid \mathcal{H}_{1}}^{-1}\left(\widetilde{\boldsymbol{\mu}}_{\mathcal{H}_{1}}-\widetilde{\boldsymbol{\mu}}_{\mathcal{H}_{0}}\right)\right\|_{2}^{2}}
$$

Once $\mathbf{f}_{\mathrm{wl}-\mathrm{opt}}$ is determined, from (23) we can set the threshold to obtain a targeted false-alarm probability $P_{f}^{\mathrm{wl}}$ :

$$
\gamma_{\mathrm{wl}-\mathrm{opt}}=Q^{-1}\left(P_{f}^{\mathrm{wl}}\right) \sqrt{\mathbf{f}_{\mathrm{wl}-\mathrm{opt}}^{H} \mathbf{C}_{\mathbf{y}_{a} \mid \mathcal{H}_{0}} \mathbf{f}_{\mathrm{wl}-\mathrm{opt}}}+\mathbf{f}_{\mathrm{wl}-\mathrm{opt}}^{H} \widetilde{\boldsymbol{\mu}}_{\mathcal{H}_{0}}
$$

\section{The Linear Cooperative Spectrum Sensing Technique}

Here, we develop the $\mathrm{L}$ combining rule for the considered RC model, by following the same guidelines of the $\mathrm{WL}$ rule. Once the FC receives the $\left\{\mathbf{y}_{i}(l)\right\}$, a $\mathrm{L}$ global test statistic $d(l)$ is calculated by linearly processing the received vector:

$$
d(l) \triangleq \mathbf{w}^{H} \mathbf{y}(l)
$$


Since the vector $\mathbf{y}(l)$ is a complex Gaussian vector, its linear combination $d(l)$ is also a complex Gaussian r.v.. In the following, the weighting vector $\mathbf{w}$ is singled out by maximizing the modified deflection coefficient:

$$
\begin{aligned}
m_{1}^{2}(\mathbf{w}) & \triangleq \frac{\left|E\left[d(l) \mid \mathcal{H}_{1}\right]-E\left[d(l) \mid \mathcal{H}_{0}\right]\right|^{2}}{\operatorname{Var}\left[d(l) \mid \mathcal{H}_{1}\right]}= \\
& =\frac{\left|\mathbf{w}^{H}\left(\boldsymbol{\mu}_{\mathcal{H}_{1}}-\boldsymbol{\mu}_{\mathcal{H}_{0}}\right)\right|^{2}}{\mathbf{w}^{H} \mathbf{C}_{\mathbf{y} \mid \mathcal{H}_{1}} \mathbf{w}}
\end{aligned}
$$

where $\boldsymbol{\mu}_{\mathcal{H}_{j}}$ and $\mathbf{C}_{\mathbf{y} \mid \mathcal{H}_{j}}$ are given by (6) and (8), respectively. The second equality in (33) is obtained by exploiting the expression (32) of $d(l)$. To avoid undetermined solutions, (33) is maximized under the unit-norm constraint for $\mathbf{w}$ :

$$
\max _{\mathbf{w}} m_{1}^{2}(\mathbf{w}) \quad \text { subject to } \mathbf{w}^{H} \mathbf{w}=1
$$

By using the Cauchy-Schwartz's inequality, the modified deflection coefficient is maximized by choosing $\mathrm{w}$ as follows:

$$
\mathbf{w}_{\text {l-opt }}=\zeta \mathbf{C}_{\mathbf{y} \mid \mathcal{H}_{1}}^{-1}\left(\boldsymbol{\mu}_{\mathcal{H}_{1}}-\boldsymbol{\mu}_{\mathcal{H}_{0}}\right)
$$

The constraint $\mathbf{w}^{H} \mathbf{w}=1$ can be satisfied by setting the constant ${ }^{5} \zeta=1 /\left\|\mathbf{C}_{\mathbf{y} \mid \mathcal{H}_{1}}^{-1}\left(\boldsymbol{\mu}_{\mathcal{H}_{1}}-\boldsymbol{\mu}_{\mathcal{H}_{0}}\right)\right\|_{2}^{2}$, obtaining so that the modified deflection coefficient is maximized by:

$$
\mathbf{w}_{\text {l-opt }}=\frac{\mathbf{C}_{\mathbf{y} \mid \mathcal{H}_{1}}^{-1}\left(\boldsymbol{\mu}_{\mathcal{H}_{1}}-\boldsymbol{\mu}_{\mathcal{H}_{0}}\right)}{\|\left.\mathbf{C}_{\mathbf{y} \mid \mathcal{H}_{1}}^{-1}\left(\boldsymbol{\mu}_{\mathcal{H}_{1}}-\boldsymbol{\mu}_{\mathcal{H}_{0}}\right)\right|_{2} ^{2}}
$$

Once $\mathbf{w}_{\text {l-opt }}$ is determined, the FC takes the global decision on the presence of the PU by comparing the real part of the optimized L test statistic with a decision threshold $\gamma_{1}$ :

$$
\operatorname{Re}[d(l)] \triangleq \operatorname{Re}\left[\mathbf{w}_{\text {l-opt }}^{H} \mathbf{y}(l)\right] \stackrel{\gtrless \mathcal{H}_{1}}{\gtrless} \gamma_{1}
$$

where the real-part operator takes into account that the information symbols $\left\{u_{i}(q)\right\}$ are real, while the linear combination $d(l)$ of the received vector is complex. Since $d(l)$ is a Gaussian r.v., its real part is also Gaussian. Hence, the false-alarm $P_{f}^{1}$ and the detection $P_{d}^{\mathrm{l}}$ probabilities can be expressed as:

$$
\begin{aligned}
& P_{f}^{\mathrm{l}} \triangleq P\left[\operatorname{Re}[d(l)]>\gamma_{1} \mid \mathcal{H}_{0}\right]=Q\left(\frac{\gamma_{1}-E\left[\operatorname{Re}[d(l)] \mid \mathcal{H}_{0}\right]}{\sqrt{\operatorname{Var}\left[\operatorname{Re}[d(l)] \mid \mathcal{H}_{0}\right]}}\right) \\
& P_{d}^{\mathrm{l}} \triangleq P\left[\operatorname{Re}[d(l)]>\gamma_{1} \mid \mathcal{H}_{1}\right]=Q\left(\frac{\gamma_{1}-E\left[\operatorname{Re}[d(l)] \mid \mathcal{H}_{1}\right]}{\sqrt{\operatorname{Var}\left[\operatorname{Re}[d(l)] \mid \mathcal{H}_{1}\right]}}\right)
\end{aligned}
$$

To make explicit the equations (38) and (39), first we derive the mean and then the variance of the r.v. $\operatorname{Re}[d(l)]$ :

$$
\begin{aligned}
\mu_{1 \mid \mathcal{H}_{j}} & \triangleq E\left[\operatorname{Re}[d(l)] \mid \mathcal{H}_{j}\right]=E\left[\frac{d(l)+d^{*}(l)}{2} \mid \mathcal{H}_{j}\right]= \\
& =\operatorname{Re}\left(\mathbf{w}_{\text {l-opt }}^{H} \boldsymbol{\mu}_{\mathcal{H}_{j}}\right)
\end{aligned}
$$

where $\boldsymbol{\mu}_{\mathcal{H}_{j}}$ is given by (6), and $\mathbf{w}_{\text {l-opt }}$ by (36). In (40) we have used the notable relation $\operatorname{Re}[z]=\left(z+z^{*}\right) / 2$. By using the equality $\operatorname{Re}^{2}(z)=\left(|z|^{2}+\operatorname{Re}\left(z^{2}\right)\right) / 2$, after some algebraic manipulations, the variance of $\operatorname{Re}[d(l)]$ is obtained:

\footnotetext{
${ }^{5}$ The unit-norm constraint allows us to determinate only the module of the constant $\zeta$. However, we can choose a real-value constant $\zeta$, since the deflection coefficient (33) is not affected by phase rotations.
}

$$
\begin{aligned}
\varphi_{1 \mid \mathcal{H}_{j}}^{2} & \triangleq \operatorname{Var}\left[\operatorname{Re}[d(l)] \mid \mathcal{H}_{j}\right]=E\left[\operatorname{Re}^{2}[d(l)] \mid \mathcal{H}_{j}\right]-\left|E\left[\operatorname{Re}[d(l)] \mid \mathcal{H}_{j}\right]\right|^{2} \\
& =\frac{\mathbf{w}_{\mathrm{l}-\mathrm{opt}}^{H} \mathbf{C}_{\mathbf{y} \mid \mathcal{H}_{j}} \mathbf{w}_{\text {l-opt }}+\operatorname{Re}\left[\mathbf{w}_{\mathrm{l}-\mathrm{opt}}^{H} \mathbf{C}_{\mathbf{y}^{*} \mid \mathcal{H}_{j}} \mathbf{w}_{\mathrm{l} \text {-opt }}^{*}\right]}{2}
\end{aligned}
$$

where $\mathbf{C}_{\mathbf{y} \mid \mathcal{H}_{j}}$ and $\mathbf{C}_{\mathbf{y}^{*} \mid \mathcal{H}_{j}}$ are given by (8) and (12), respectively. Consequently, by taking into account (40) and (41), $P_{f}^{\mathrm{l}}$ and $P_{d}^{\mathrm{l}}$ in (38) and (39) can be rewritten as:

$$
\begin{aligned}
P_{f}^{1} & =Q\left(\frac{\gamma_{1}-\mu_{1 \mid \mathcal{H}_{0}}}{\sqrt{\varphi_{1 \mid \mathcal{H}_{0}}^{2}}}\right)= \\
& =Q\left(\frac{\sqrt{2}\left(\gamma_{1}-\operatorname{Re}\left[\mathbf{w}_{1-\text { opt }}^{H} \boldsymbol{\mu}_{\mathcal{H}_{0}}\right]\right)}{\sqrt{\mathbf{w}_{\text {l-opt }}^{H} \mathbf{C}_{\mathbf{y} \mid \mathcal{H}_{0}} \mathbf{w}_{\text {l-opt }}+\operatorname{Re}\left[\mathbf{w}_{\text {l-opt }}^{H} \mathbf{C}_{\mathbf{y}^{*} \mid \mathcal{H}_{0}} \mathbf{w}_{\text {l-opt }}^{*}\right]}}\right) \\
P_{d}^{1} & =Q\left(\frac{\gamma_{1}-\mu_{1 \mid \mathcal{H}_{1}}}{\sqrt{\varphi_{1 \mid \mathcal{H}_{1}}^{2}}}\right)= \\
& =Q\left(\frac{\sqrt{2}\left(\gamma_{1}-\operatorname{Re}\left[\mathbf{w}_{\text {l-opt }}^{H} \boldsymbol{\mu}_{\mathcal{H}_{1}}\right]\right)}{\sqrt{\mathbf{w}_{\text {l-opt }}^{H} \mathbf{C}_{\mathbf{y} \mid \mathcal{H}_{1}} \mathbf{w}_{\text {l-opt }}+\operatorname{Re}\left[\mathbf{w}_{\text {l-opt }}^{H} \mathbf{C}_{\mathbf{y}^{*} \mid \mathcal{H}_{1}} \mathbf{w}_{\text {l-opt }}^{*}\right]}}\right)
\end{aligned}
$$

From (42) we can set the threshold $\gamma_{1}$ to obtain a targeted $P_{f}^{1}$ :

$$
\begin{aligned}
\gamma_{\text {l-opt }} & =\frac{Q^{-1}\left(P_{f}^{1}\right) \sqrt{\mathbf{w}_{\text {l-opt }}^{H} \mathbf{C}_{\mathbf{y} \mid \mathcal{H}_{0}} \mathbf{w}_{\text {l-opt }}+\operatorname{Re}\left[\mathbf{w}_{\text {l-opt }}^{H} \mathbf{C}_{\mathbf{y}^{*} \mid \mathcal{H}_{0}} \mathbf{w}_{\text {l-opt }}^{*}\right]}}{\sqrt{2}} \\
& +\operatorname{Re}\left(\mathbf{w}_{\text {l-opt }}^{H} \boldsymbol{\mu}_{\mathcal{H}_{0}}\right)
\end{aligned}
$$

In the following, we prove that the deflection coefficient assured by the proposed WL technique is greater than the one assured by the proposed $\mathrm{L}$ technique. Since a greater deflection coefficient corresponds to a major detection sensitivity [8], [15], [21] in the gaussian hypothesis, the WL technique outperforms the $\mathrm{L}$ one, as confirmed by the numerical results in Section V-A. We observe that the maximized deflection coefficients assured by the optimized vectors $\mathbf{f}_{\mathrm{wl}-\text { opt }}(30)$ and $\mathbf{w}_{\text {l-opt }}(36)$ are equal to, respectively:

$$
\begin{aligned}
m_{\text {wl-opt }}^{2}\left(\mathbf{f}_{\text {wl-opt }}\right) & =\left(\widetilde{\boldsymbol{\mu}}_{\mathcal{H}_{1}}-\widetilde{\boldsymbol{\mu}}_{\mathcal{H}_{0}}\right)^{H} \mathbf{C}_{\mathbf{y}_{a} \mid \mathcal{H}_{1}}^{-1}\left(\widetilde{\boldsymbol{\mu}}_{\mathcal{H}_{1}}-\widetilde{\boldsymbol{\mu}}_{\mathcal{H}_{0}}\right) \\
m_{\text {l-opt }}^{2}\left(\mathbf{w}_{\text {l-opt }}\right) & =\left(\boldsymbol{\mu}_{\mathcal{H}_{1}}-\boldsymbol{\mu}_{\mathcal{H}_{0}}\right)^{H} \mathbf{C}_{\mathbf{y} \mid \mathcal{H}_{1}}^{-1}\left(\boldsymbol{\mu}_{\mathcal{H}_{1}}-\boldsymbol{\mu}_{\mathcal{H}_{0}}\right)
\end{aligned}
$$

Proposition 1: The Widely-Linear deflection coefficient (45) is greater than the Linear deflection coefficient (46), if the reporting channels are modeled as temporal dispersive.

$$
\text { Proof: See Appendix A }
$$

\section{TheORETICAL COMPARISON OF THE WL AND L COOPERATIVE TECHNiQues}

In this section, we prove that the $\mathrm{L}$ and the WL fusion schemes perform exactly the same, when each RC between the FC and the CR user is modeled as multipath frequency non-selective channel. Therefore, when such assumption holds (e.g., when the channel delay spread is significantly less than the CR symbol duration), there is not anymore advantage to use the WL fusion scheme, which exhibits an higher, although limited, computational complexity than the L one. Preliminarily, we 
note that, when the RC is modeled as a frequency nonselective channel, its complex lowpass equivalent response appears as a multiplicative factor, i.e., $L_{g_{i}}=1$ in (3). Therefore, in the following, first we modify the expressions of the quantities presented in Section II-B according to the simplified channel model, keeping unchanged the hypotheses drawn in the previous sections. Then, we carry out the theoretical analysis.

The base-band signal (3) at the RF front-end of the FC received from the $i$-th $\mathrm{CR}$ user can be rewritten as:

$$
y_{i}(l)=h_{i} u_{i}(l)+n_{i}(l), \quad i \in\{1, \ldots, M\}
$$

Since there is no need to jointly elaborate $L_{e}$ consecutive received samples for each $\mathrm{CR}$ user, the expression (5) of the collected received vector $\mathbf{y}(l)$ is simplified as follows:

$$
\begin{aligned}
\mathbf{y}(l) \triangleq & \triangleq c_{y_{1}(l)}^{\left[\begin{array}{c}
y_{2}(l) \\
\vdots \\
y_{M}(l)
\end{array}\right]}=\underbrace{\left[\begin{array}{cccc}
h_{1} & 0 & \ldots & 0 \\
0 & h_{2} & \ldots & 0 \\
\vdots & \vdots & \ddots & \vdots \\
0 & 0 & \ldots & h_{M}
\end{array}\right]}_{\triangleq \mathbf{H} \in \mathbb{C}^{M} \times M} \underbrace{\left[\begin{array}{c}
u_{1}(l) \\
u_{2}(l) \\
\vdots \\
u_{M}(l)
\end{array}\right]}_{\triangleq \mathbf{u}(l) \in \mathbb{R}^{M}}+ \\
& \overbrace{\left[\begin{array}{c}
n_{1}(l) \\
n_{2}(l) \\
\vdots \\
n_{M}(l)
\end{array}\right]}^{\left[\mathcal{C}^{M}\right.}=\mathbf{H} \mathbf{u}(l)+\mathbf{n}(l)
\end{aligned}
$$

Therefore, taking into account the relations (2) and (48), the mean in (6) and the covariance matrix in (8) of the complex Gaussian random vector $\mathbf{y}(l)$ can be rewritten as:

$$
\boldsymbol{\mu}_{\mathcal{H}_{j}}=E\left[\mathbf{y}(l) \mid \mathcal{H}_{j}\right]=\mathbf{H} E\left[\mathbf{u}(l) \mid \mathcal{H}_{j}\right]=\mathbf{H} \boldsymbol{\eta}_{\mathcal{H}_{j}} \in \mathbb{C}^{M}
$$

where

$$
\begin{aligned}
& \boldsymbol{\eta}_{\mathcal{H}_{j}} \triangleq E\left[\mathbf{u}(l) \mid \mathcal{H}_{j}\right]= \\
& = \begin{cases}{\left[N \sigma_{1}^{2} \ldots N \sigma_{M}^{2}\right]^{T}} & \mathcal{H}_{0} \\
{\left[\left(E_{s}\left|g_{1}\right|^{2}+N \sigma_{1}^{2}\right) \ldots\left(E_{s}\left|g_{M}\right|^{2}+N \sigma_{M}^{2}\right)\right]^{T}} & \mathcal{H}_{1}\end{cases} \\
& \mathbf{C}_{\mathbf{y} \mid \mathcal{H}_{j}} \triangleq E\left[(\mathbf{y}(l)-\boldsymbol{\mu})(\mathbf{y}(l)-\boldsymbol{\mu})^{H} \mid \mathcal{H}_{j}\right] \in \mathbb{C}^{M \times M}= \\
& \quad=\mathbf{H} \mathbf{C}_{\mathbf{u} \mid \mathcal{H}_{j}} \mathbf{H}^{H}+\mathbf{R}_{\mathbf{n}}
\end{aligned}
$$

The noise covariance matrix $\mathbf{R}_{\mathbf{n}}$ and the transmitted statistics covariance matrix $\mathbf{C}_{\mathbf{u} \mid \mathcal{H}_{j}}$ are equal to:

$$
\begin{aligned}
& \mathbf{R}_{\mathbf{n}}=\operatorname{diag}\left[\delta_{1}^{2} \delta_{2}^{2} \ldots \delta_{M}^{2}\right] \in \mathbb{R}^{M \times M} \\
& \mathbf{C}_{\mathbf{u} \mid \mathcal{H}_{j}}=\operatorname{diag}\left[\zeta_{1 \mid \mathcal{H}_{j}} \zeta_{2 \mid \mathcal{H}_{j}} \ldots \zeta_{M \mid \mathcal{H}_{j}}\right] \in \mathbb{R}^{M \times M}
\end{aligned}
$$

where $\zeta_{i \mid \mathcal{H}_{j}}$ denotes the $\operatorname{Var}\left[u_{i}(q) \mid \mathcal{H}_{j}\right]$ given by (2). Finally, the pseudo-covariance matrix of $\mathbf{y}(l)$ is equal to:

$$
\begin{aligned}
\mathbf{C}_{\mathbf{y}^{*} \mid \mathcal{H}_{j}} & \triangleq E\left[(\mathbf{y}(l)-\boldsymbol{\mu})(\mathbf{y}(l)-\boldsymbol{\mu})^{T} \mid \mathcal{H}_{j}\right] \in \mathbb{C}^{M \times M}= \\
& =\mathbf{H C}_{\mathbf{u} \mid \mathcal{H}_{j}} \mathbf{H}^{T}
\end{aligned}
$$

with $\mathbf{C}_{\mathbf{u} \mid \mathcal{H}_{j}}$ given in (52). We underline that all the previous matrices are diagonal as a consequence of the simplified system model, unlike the matrices in Section II.

Since the theoretical derivations in Sections III-B and III-C are blind with respect to the adopted $\mathrm{RC}$ model, the relations
(30) and (36) continue to hold provided that the synthetic statistical parameters are those given in (49), (51) and (53). In the following, for the sake of clarity, we report the closed-form expressions of the $\mathrm{L}$ and $\mathrm{WL}$ rules:

$$
\begin{gathered}
\mathbf{w}_{\text {l-opt }}=\frac{\mathbf{C}_{\mathbf{y} \mid \mathcal{H}_{1}}^{-1}\left(\boldsymbol{\mu}_{\mathcal{H}_{1}}-\boldsymbol{\mu}_{\mathcal{H}_{0}}\right)}{\left\|\mathbf{C}_{\mathbf{y} \mid \mathcal{H}_{1}}^{-1}\left(\boldsymbol{\mu}_{\mathcal{H}_{1}}-\boldsymbol{\mu}_{\mathcal{H}_{0}}\right)\right\|_{2}^{2}} \\
\mathbf{f}_{\text {wl-opt }} \triangleq\left[\begin{array}{l}
\mathbf{f}_{1, \mathrm{wl} \text {-opt }} \\
\mathbf{f}_{1, \mathrm{wl} \text {-opt }}^{*}
\end{array}\right]=\frac{\mathbf{C}_{\mathbf{y}_{a} \mid \mathcal{H}_{1}}^{-1}\left(\widetilde{\boldsymbol{\mu}}_{\mathcal{H}_{1}}-\widetilde{\boldsymbol{\mu}}_{\mathcal{H}_{0}}\right)}{\left\|\mathbf{C}_{\mathbf{y}_{a} \mid \mathcal{H}_{1}}^{-1}\left(\widetilde{\boldsymbol{\mu}}_{\mathcal{H}_{1}}-\widetilde{\boldsymbol{\mu}}_{\mathcal{H}_{0}}\right)\right\|_{2}^{2}}
\end{gathered}
$$

In (55), according to (17) and (19), we have that

$$
\begin{gathered}
\widetilde{\boldsymbol{\mu}}_{\mathcal{H}_{j}}=\left[\begin{array}{c}
\mathbf{H} \\
\mathbf{H}^{*}
\end{array}\right] \boldsymbol{\eta}_{\mathcal{H}_{j}}=\mathbf{H}_{a} \boldsymbol{\eta}_{\mathcal{H}_{j}} \\
\mathcal{C}_{\mathbf{y}_{a} \mid \mathcal{H}_{j}}=\left[\begin{array}{cc}
\mathbf{C}_{\mathbf{y} \mid \mathcal{H}_{j}} & \mathbf{C}_{\mathbf{y}^{*} \mid \mathcal{H}_{j}} \\
\mathbf{C}_{\mathbf{y}^{*} \mid \mathcal{H}_{j}}^{*} & \mathbf{C}_{\mathbf{y} \mid \mathcal{H}_{j}}^{*}
\end{array}\right]
\end{gathered}
$$

Before to address the theoretical comparison of the WL and L schemes through Theorem 1, we present in Proposition 2 an intermediate result.

Proposition 2: The WL-subvector $\mathbf{f}_{1, \mathrm{wl}-\mathrm{opt}}$ given in (55) can be expressed as:

$$
\mathbf{f}_{1, \mathrm{wl} \text {-opt }}=\frac{\left[\mathbf{I}+\mathbf{C}_{\mathbf{y} \mid \mathcal{H}_{1}}^{-1} \mathbf{C}_{\mathbf{y}^{*} \mid \mathcal{H}_{1}} \mathbf{H}^{-1} \mathbf{H}^{H}\right]^{-1} \mathbf{w}_{\text {l-opt }}}{\sqrt{2} \|\left.\left[\mathbf{I}+\mathbf{C}_{\mathbf{y} \mid \mathcal{H}_{1}}^{-1} \mathbf{C}_{\mathbf{y}^{*} \mid \mathcal{H}_{1}} \mathbf{H}^{-1} \mathbf{H}^{H}\right]^{-1} \mathbf{w}_{\text {l-opt }}\right|_{2} ^{2}}
$$

Proof: See Appendix B.

Since $\left[\mathbf{I}+\mathbf{C}_{\mathbf{y} \mid \mathcal{H}_{1}}^{-1} \mathbf{C}_{\mathbf{y}^{*} \mid \mathcal{H}_{1}} \mathbf{H}^{-1} \mathbf{H}^{H}\right]^{-1}$ in (58) is a diagonal matrix for the adopted channel model, $\mathbf{f}_{1, \mathrm{wl} \text {-opt }}$ is a scaled version of the linear vector $\mathrm{w}_{\mathrm{l}-\mathrm{opt}}$ given in (54).

Theorem 1: The detection probability assured by the WL detector for a fixed false-alarm probability $P_{f}$ is the same of the $\mathrm{L}$ detector, provided that the $\mathrm{CR}$ user transmissions are orthogonal, and that each link between the FC and the CR users is modeled as multipath frequency nonselective channel.

Proof: To prove that the detection probabilities of the WL and $\mathrm{L}$ detectors are the same, we prove that the arguments of the Q-functions in (24) and (43) are equal. At this end, taking into account the closed-form expression (31) of the decision threshold for the WL cooperative technique, we can rewrite the argument of the Q-function in (24) as in (59), reported at the top of the next page. The last equality in (59) follows from (57). Analogously, by substituting (44) in the argument of the Q-function (43) for the $\mathrm{L}$ case, one has the result (60), reported at the top of the next page. By comparing (59) and (60), we recognize that they are equal provided that $\mathbf{f}_{1 \text {,wl-opt }}$ and $\mathbf{w}_{\text {l-opt }}$ are scaled versions of each other. Hence, by accounting for Proposition 2, the theorem is proved.

In the particular case of high SNR region, the scaled factor, which allows to express $\mathbf{f}_{1 \text {,wl-opt }}$ as function of the linear $\mathbf{w}_{\text {l-opt }}$, has a very simple expression thanks to the following corollary:

Corollary 1: In the high-SNR region at the FC side, the WL-subvector $\mathbf{f}_{1, \text { wl-opt }}$ is ${ }^{6}$ :

$$
\lim _{\left\{\delta_{i}^{2} \rightarrow 0\right\}_{i=1}^{M}} \mathbf{f}_{1, \mathrm{wl} \text {-opt }}=\frac{1}{\sqrt{2}} \mathbf{w}_{\text {l-opt }}
$$

\footnotetext{
${ }^{6}$ The coefficient $1 / \sqrt{2}$ in (61) is intuitively justified by considering that $\mathbf{f}_{\mathrm{wl}-\text { opt }}$ is designed with the unit-norm constraint.
} 


$$
\begin{aligned}
& \frac{\gamma_{\mathrm{wl}}-\mathbf{f}_{\mathrm{wl}-\mathrm{opt}}^{H} \widetilde{\boldsymbol{\mu}}_{\mathcal{H}_{1}}}{\sqrt{\mathbf{f}_{\mathrm{wl}-\mathrm{opt}}^{H} \mathbf{C}_{\mathbf{y}_{a} \mid \mathcal{H}_{1}} \mathbf{f}_{\mathrm{wl}-\mathrm{opt}}}}=\frac{Q^{-1}\left(P_{f}\right) \sqrt{\mathbf{f}_{\mathrm{wl}-\mathrm{opt}}^{H} \mathbf{C}_{\mathbf{y}_{a} \mid \mathcal{H}_{0}} \mathbf{f}_{\mathrm{wl}-\mathrm{opt}}}+\mathbf{f}_{\mathrm{wl}-\mathrm{opt}}^{H}\left(\widetilde{\boldsymbol{\mu}}_{\mathcal{H}_{0}}-\widetilde{\boldsymbol{\mu}}_{\mathcal{H}_{1}}\right)}{\sqrt{\mathbf{f}_{\mathrm{wl}-\mathrm{opt}}^{H} \mathbf{C}_{\mathbf{y}_{a} \mid \mathcal{H}_{1}} \mathbf{f}_{\mathrm{wl}-\mathrm{opt}}}}= \\
& \frac{Q^{-1}\left(P_{f}\right) \sqrt{\mathbf{f}_{1, \mathrm{wl}-\mathrm{opt}}^{H} \mathbf{C}_{\mathbf{y} \mid \mathcal{H}_{0}} \mathbf{f}_{1, \mathrm{wl}-\mathrm{opt}}+\operatorname{Re}\left[\mathbf{f}_{1, \mathrm{wl}-\mathrm{opt}}^{H} \mathbf{C}_{\mathbf{y}^{*} \mid \mathcal{H}_{0}} \mathbf{f}_{1, \mathrm{wl}-\mathrm{opt}}^{*}\right]}+\sqrt{2} \operatorname{Re}\left[\mathbf{f}_{1, \mathrm{wl}-\mathrm{opt}}^{H} \mathbf{H}\left(\boldsymbol{\eta}_{\mathcal{H}_{0}}-\boldsymbol{\eta}_{\mathcal{H}_{1}}\right)\right]}{\sqrt{\mathbf{f}_{1, \mathrm{wl}-\mathrm{opt}}^{H} \mathbf{C}_{\mathbf{y} \mid \mathcal{H}_{1}} \mathbf{f}_{1, \mathrm{wl}-\mathrm{opt}}+\operatorname{Re}\left[\mathbf{f}_{1, \mathrm{wl}-\mathrm{opt}}^{H} \mathbf{C}_{\mathbf{y}^{*} \mid \mathcal{H}_{1}} \mathbf{f}_{1, \mathrm{wl}-\mathrm{opt}}^{*}\right]}} \\
& \frac{\sqrt{2}\left(\gamma-\operatorname{Re}\left[\mathbf{w}_{\text {l-opt }}^{H} \boldsymbol{\mu}_{\mathcal{H}_{1}}\right]\right)}{\sqrt{\mathbf{w}_{\text {l-opt }}^{H} \mathbf{C}_{\mathbf{y} \mid \mathcal{H}_{1}} \mathbf{w}_{\text {l-opt }}+\operatorname{Re}\left[\mathbf{w}_{\text {l-opt }}^{H} \mathbf{C}_{\mathbf{y}^{*} \mid \mathcal{H}_{1}} \mathbf{w}_{\text {l-opt }}^{*}\right]}}= \\
& \frac{Q^{-1}\left(P_{f}\right) \sqrt{\mathbf{w}_{\text {l-opt }}^{H} \mathbf{C}_{\mathbf{y} \mid \mathcal{H}_{0}} \mathbf{w}_{\text {l-opt }}+\operatorname{Re}\left[\mathbf{w}_{\text {l-opt }}^{H} \mathbf{C}_{\mathbf{y}^{*} \mid \mathcal{H}_{0}} \mathbf{w}_{\text {l-opt }}^{*}\right]}+\sqrt{2} \operatorname{Re}\left[\mathbf{w}_{\text {l-opt }}^{H} \mathbf{H}\left(\boldsymbol{\eta}_{\mathcal{H}_{0}}-\boldsymbol{\eta}_{\mathcal{H}_{1}}\right)\right]}{\sqrt{\mathbf{w}_{\text {l-opt }}^{H} \mathbf{C}_{\mathbf{y} \mid \mathcal{H}_{1}} \mathbf{w}_{\text {sl-opt }}+\operatorname{Re}\left[\mathbf{w}_{\text {l-opt }}^{H} \mathbf{C}_{\mathbf{y}^{*} \mid \mathcal{H}_{1}} \mathbf{w}_{\text {l-opt }}^{*}\right]}}
\end{aligned}
$$

Proof: When the FC noise powers go to zero, i.e. $\delta_{i}^{2} \rightarrow 0, \forall i \in\{1, \ldots, M\}, \mathbf{C}_{\mathbf{y} \mid \mathcal{H}_{1}}$ in (51) becomes: $\mathbf{C}_{\mathbf{y} \mid \mathcal{H}_{1}}=$ $\mathbf{H ~ C}_{\mathbf{u} \mid \mathcal{H}_{1}} \mathbf{H}^{H}+\mathbf{R}_{\mathbf{n}} \rightarrow \mathbf{H C}_{\mathbf{u} \mid \mathcal{H}_{1}} \mathbf{H}^{H}$, hence (58) can be simplified as:

$$
\begin{aligned}
& \lim _{\left\{\delta_{i}^{2} \rightarrow 0\right\}_{i=1}^{M}} \mathbf{f}_{1, \mathrm{wl} \text {-opt }}= \\
& =\lim _{\left\{\delta_{i}^{2} \rightarrow 0\right\}_{i=1}^{M}}\left\{\frac{\left[\mathbf{I}+\mathbf{C}_{\mathbf{y} \mid \mathcal{H}_{1}}^{-1} \mathbf{C}_{\mathbf{y}^{*} \mid \mathcal{H}_{1}} \mathbf{H}^{-1} \mathbf{H}^{H}\right]^{-1} \mathbf{w}_{\text {l-opt }}}{\sqrt{2}\left\|\left[\mathbf{I}+\mathbf{C}_{\mathbf{y} \mid \mathcal{H}_{1}}^{-1} \mathbf{C}_{\mathbf{y}^{*} \mid \mathcal{H}_{1}} \mathbf{H}^{-1} \mathbf{H}^{H}\right]^{-1} \mathbf{w}_{\text {l-opt }}\right\|_{2}^{2}}\right\} \\
& =\frac{[2 \mathbf{I}]^{-1} \mathbf{w}_{\text {l-opt }}}{\sqrt{2}\left\|[2 \mathbf{I}]^{-1} \mathbf{w}_{\text {l-opt }}\right\|_{2}^{2}}=\frac{1}{\sqrt{2}} \mathbf{w}_{\text {l-opt }}
\end{aligned}
$$

By using (61) in (59), it is evident that the detection probabilities of both WL and L strategies are equal, proving the validity of Theorem 1 also in the high-SNR region.

\section{NumERICAL RESUlts}

In Subsection V-A, the performances of the proposed schemes are evaluated when the RCs are temporal dispersive. The results confirm that the WL detector outperforms the $\mathrm{L}$ one in operative conditions of practical interest. Subsection V-B validates the theoretical analysis of Section IV, by evaluating the performances of the proposed schemes when the RCs are not temporal dispersive. In both the subsections, the considered detectors are: the WL, the L, the optimal Neyman-Pearson test at the FC side (LRT) [15], and the non-cooperative strategy ( $\mathrm{NC}$ ), in which a $\mathrm{CR}$ user takes an autonomous decision based only on its local energy.

\section{A. Temporal Dispersive Reporting Channels}

Each experiment consists of $10^{6}$ independent Monte Carlo runs, and in each run the noise samples and the SCs $\left\{g_{i}\right\}_{i=1}^{M}$ are randomly and independently generated according to a zeromean complex normal distribution. For the sake of simplicity, the PU signal is assumed equal to $s(k)=1$. The lengths of the FIR filters, modeling the RCs, are set equal to $L_{g}=2$ and the tap values are randomly and independently generated according to a zero-mean complex Gaussian process. The instantaneous SNR at each CR user is defined as [5], [6], [8]: $\left\{\mathrm{SNR}_{i}\right\}_{i=1}^{M}=\left|g_{i}\right|^{2} E s /\left(N \sigma_{i}^{2}\right)$.
Experiment 1: Fig. 3 shows the Missing-Detection Probability $P_{m}$ versus (vs) the average CR user SNR, $\overline{S N R}$, for a false-alarm probability $P_{f}$ equal to $P_{f}=10^{-2}, N=50$ and the number of cooperative $\mathrm{CR}$ users equal to $M=4$. Two different values of $L_{e}$ are considered, i.e. $L_{e}=1$ and $L_{e}=3$, to evaluate the impact of the number of consecutive received samples processed by the FC on the proposed schemes. The results are obtained by considering at the $\mathrm{FC}$ different noise variance values for each $\mathrm{CR}$ user, i.e., $\delta_{1}^{2}=0.5, \delta_{2}^{2}=1$, $\delta_{3}^{2}=1.5$, and $\delta_{4}^{2}=2$, to account for different CR user sensing capabilities, conferring so generality to the analysis. The results show that the NC strategy, which is independent from $L_{e}$, performs notably worse than the cooperative ones, as expected. Instead, the performances of the proposed schemes increase when $L_{e}$ increases. Specifically, for both the $L_{e}$ values, the $\mathrm{WL}$ detector outperforms the $\mathrm{L}$ one in operative conditions of practical interest, i.e., low $\overline{S N R}$, assuring so less interference to the PU. The WL gain on the $\mathrm{L}$ detector is more pronounced when $L_{e}=1$, which corresponds to the shorter processing delay. The WL gain is justified by its capacity to exploit the information contained in the statistical pseudocovariance of the received signal. In addition, for both the $L_{e}$ values, the WL detector performs almost comparable with the optimal LRT, but with a significantly lower computational complexity.

Experiment 2: Fig. 4 shows $P_{m}$ vs $P_{f}$, for $M=4$, $L_{e}=1$ and $N=50 .\left\{\delta_{i}^{2}\right\}_{i=1}^{M}$ and $\left\{\overline{S N R}_{i}\right\}_{i=1}^{M}$ are different for each CR user in order to analyze how different sensing capabilities can affect the global decision. Specifically, the results of Fig. 4 are obtained by setting $\overline{S N R}_{1}=-8 \mathrm{~dB}$, $\overline{S N R}_{2}=-8.7 \mathrm{~dB}, \overline{S N R}_{3}=-9.3 \mathrm{~dB}, \overline{S N R}_{4}=-10$, and $\left\{\delta_{i}^{2}\right\}_{i=1}^{M}$ as in Experiment 1 . Since the CR users are characterized by different values of $\overline{S N R}$, we consider an additional non-cooperative detection strategy, referred to as NC-max, which is based on the decision of the CR user characterized by the maximum SNR. In such a way, we show that a non-cooperative strategy performs notably worse than the cooperative ones, even if the decision on the presence of the PU is taken by the CR user that experiences the best channel condition as shown in Fig. 4. The results confirm also the capacity of the WL technique to outperform the $\mathrm{L}$ one in 

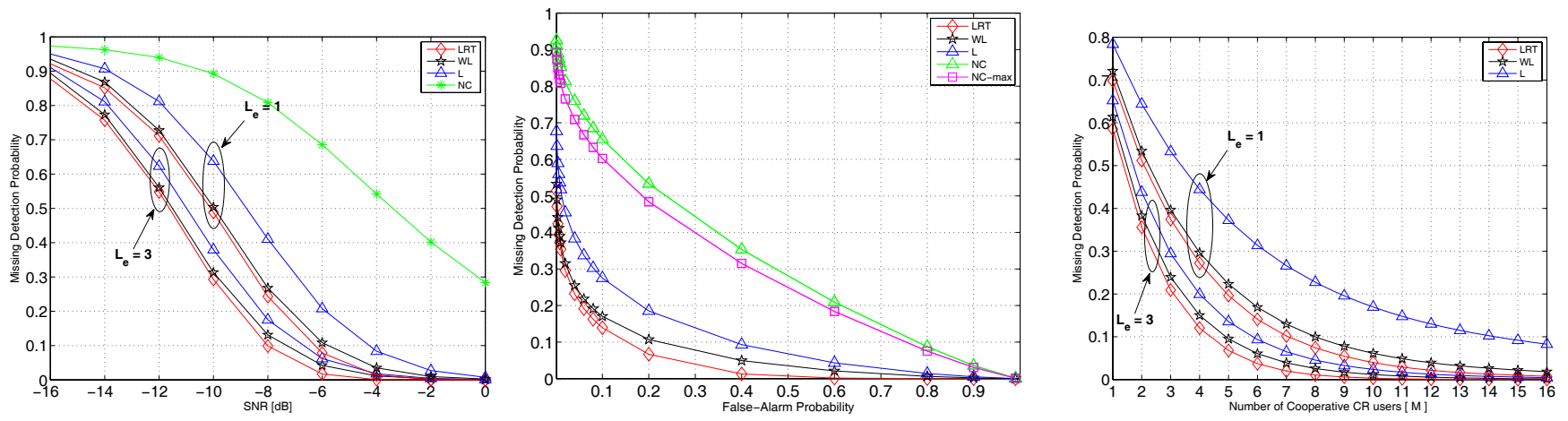

Fig. 3. $\quad P_{m}$ vs SNR, for $P_{f}=10^{-2}$ and $M=4$ Fig. 4. $\quad P_{m}$ vs $P_{f}$, for $L_{e}=1$ and $M=4$

Fig. 5. $P_{m}$ vs $M$, for $P_{f}=10^{-2}$
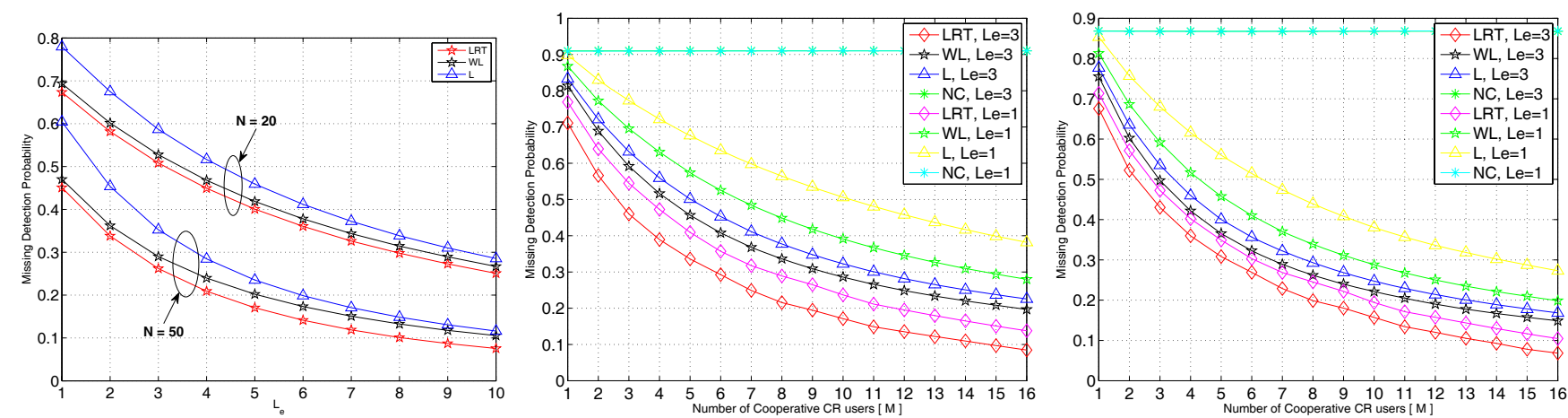

Fig. 6. $P_{m}$ vs $L_{e}$, for $P_{f}=10^{-2}$ and $M=4$ Fig. 7. $\quad P_{m}$ vs $M$, for $\Delta=0.5 \mathrm{~dB}$ and $N=50$ Fig. 8 . $P_{m}$ vs $M$, for $\Delta=0.5 \mathrm{~dB}$ and $N=100$

presence of different sensing capabilities.

Experiment 3: Here, $P_{m}$ is evaluated vs the number $\mathrm{M}$ of cooperative CR users, for $P_{f}=10^{-2}, N=50$ and for two different values of $L_{e}$, i.e. $L_{e}=1$ and $L_{e}=3$. In this and in the next experiment, the NC strategy is not considered since its performances do not depend on the parameters $M$ and $L_{e}$. The CR users are characterized by different values of $\left\{\overline{S N R}_{i}\right\}_{i=1}^{M}$ and $\left\{\delta_{i}^{2}\right\}_{i=1}^{M}$, set as follows: the average SNR value is equal to $-8 \mathrm{~dB}$ when $M=1$ and then it decreases, with step $0.2 \mathrm{~dB}$, every time the a new CR user is considered. For example, when $M=16,\left\{\overline{S N R}_{i}\right\}_{i=1}^{M}$ are set equal to: $\overline{S N R}_{1}=-8 \mathrm{~dB}, \overline{S N R}_{2}=-8.2 \mathrm{~dB}, \ldots, \overline{S N R}_{16}=-11$ $\mathrm{dB}$. Similarly, the noise variance at the $\mathrm{FC}$ is set equal to 0.2 when $M=1$, and then it increases with step 0.1 every time that a new CR user is considered. As shown in Fig. 5, the sensing reliability improves as $M$ increases, even if the average local SNR decreases and the noise variance at the FC increases when a new $\mathrm{CR}$ user is involved in the cooperative decision. Moreover, when $L_{e}$ increases, it is possible to reduce the number of cooperative $\mathrm{CR}$ users to achieve targeted performances, reducing so the coordination cost in terms of network overhead. However, if $L_{e}$ increases, the delay processing increases as well, and hence there is a trade-off. Finally, the effectiveness of the proposed WL technique, which is able to perform almost comparable with the LRT, is confirmed.

Experiment 4: Fig. 6 shows $P_{m}$ vs the number of samples $L_{e}$ jointly processed by the $\mathrm{FC}$, for $P_{f}=10^{-2}, M=4$ and for different values of $N$, i.e., $N=20$ and $N=50$. $\left\{\overline{S N R}_{i}\right\}_{i=1}^{M}$ are set equal to $\overline{S N R}_{1}=-8 \mathrm{~dB}, \overline{S N R}_{2}=-9.3$
$\mathrm{dB}, \overline{S N R}_{3}=-10.6 \mathrm{~dB}, \overline{S N R}_{4}=-12 \mathrm{~dB}$, and $\left\{\delta_{i}^{2}\right\}_{i=1}^{M}$ as in Experiment 1. When $L_{e}$ increases, it is possible to reduce $N$ for achieving targeted performances, reducing so the sensing time. However, if $L_{e}$ increases, the FC delay processing increases as well, and therefore there is a tradeoff also in this case. Finally, the effectiveness of the WL rule is confirmed as well.

Experiment 5: We evaluate the impact of the noise uncertainty problem (NUP) [22], [23], on the performance of the proposed schemes. The NUP affects the performance of the energy detector that below a certain SNR, called $S N R_{\text {wall }}$, fails to detect the PU signal. We evaluate $P_{m}$ vs $M$ for different values of $L_{e}$, i.e. $L_{e}=1$ and $L_{e}=3$, when the noise uncertainty $\Delta$ is set equal to a common used value, i.e. $\Delta=0.5 \mathrm{~dB},[22]$, [23]. $\overline{S N R}$ is equal to $-8 \mathrm{~dB}$, notable smaller than the $S N R_{\text {wall }}$ corresponding to $\Delta$ [22], [23]. The CR users are characterized by different values of $\left\{\delta_{i}^{2}\right\}_{i=1}^{M}$, set as in Experiment 3. As shown in Fig. 7, the energy detector fails to detect the PU as expected (the NC curve exhibits a $P_{m} \simeq 0.9$ ). However, we can improve the sensing reliability of the energy detector by resorting to the proposed detectors. In fact, although the performances of the WL, L and LRT schemes are smaller than those in absence of the NUP, they increase as $M$ increases, even if $\overline{S N R}$ is smaller than $S N R_{\text {wall }}$ and even if the noise variance $\delta_{i}^{2}$ at the FC increases when a new CR user is involved in the cooperation. Moreover, Fig. 7 shows also that it is possible to reduce the value of $M$ needed to achieve targeted performances, by increasing $L_{e}$. The same considerations hold with reference to Fig. 8 , obtained for $N=100$. Fig. 8 
shows that the energy detector cannot overcome the NUP by simply increasing the sensing time. In fact, the NC curve exhibits again a $P_{m} \simeq 0.9$. Instead, the sensing reliability of the proposed detectors increases when $N$ increases as well. Hence, the proposed schemes can overcome the NUP, since they exploit the freedom degrees represented by the number of cooperative CR users, the number of samples $L_{e}$ processed by the FC, the sensing length $N$.

\section{B. No-Temporal Dispersive Reporting Channels}

Experiment 6: Fig. 9 shows the detection probability $P_{d}$ vs the average SNR, with the same simulation setting of Experiment 1, but now $L_{g}=L_{e}=1$, since the RCs are modeled as multipath frequency non-selective channels. Since the curves of the WL and L detectors practically coincide, the validity of Theorem 1 is confirmed. Plus, the NC strategy performs notably worse than the cooperative ones, as already observed in Experiment 1. The optimal LRT outperforms the proposed ones, as expected, but with a negligible gain.

Experiment 7: In this experiment we evaluate $P_{d}$ vs $P_{f}$ with the same simulation setting of Experiment 2. Fig. 10 shows that the $\mathrm{WL}$ and $\mathrm{L}$ detectors perform the same, confirming again the validity of Theorem 1 . Moreover, we note that as $P_{f}$ increases, $P_{d}$ increases as well, although larger $P_{f}$ results in lower CR user spectrum efficiency. For $P_{f}$ values of practical interest, i.e. $P_{f} \leq 10^{-1}$, the proposed schemes perform practically the same of the optimal LRT, with a computational complexity significantly lower.

Experiment 8: Finally, in this experiment we evaluate $P_{d}$ vs the number $M$ of $\mathrm{CR}$ users, with the same simulation setting of Experiment 3. Fig. 11 shows that the WL and the $\mathrm{L}$ performance curves coincide practically, confirming again the validity of Theorem 1 . Moreover, we can observe that, when $M$ increases, the reliability of spectrum sensing increases as well, even if the average CR user SNR decreases, as underlined in Experiment 3. Therefore the interference against the primary network decreases, as the number $\mathrm{M}$ of $\mathrm{CR}$ user increases.

\section{CONCLUSiOnS}

In this paper, the effects of temporal dispersive reporting channels (RCs) on cooperative spectrum sensing have been investigated by proposing two optimized fusion techniques: the Widely Linear (WL) and the Linear (L). For both the schemes closed-form expressions of the detection and falsealarm probabilities are provided, and numerical results show that the WL detector outperforms the $\mathrm{L}$ one in operative conditions of practical interest. Moreover, the WL detector performs almost comparable with the optimal LRT-based fusion rule, but with a significantly lower computational complexity. Finally, a theoretical comparison between the two proposed techniques has been carried out, proving their performance equivalence under the assumptions of orthogonal transmissions among the cognitive radio users and RCs modeled as multipath frequency non-selective channels. The theoretical analysis has been validated numerically. In future, we would like to explore the effects of temporal dispersive reporting channels on other data fusion algorithms, such as cyclostationary detection, spacetime combining, and eigenvalue-based detection.

\section{APPENDIX A}

PROOF OF PROPOSITION 1

We provide the analytical proof in the simplified case of one cooperative $\mathrm{CR}$ user, i.e. $M=1$, and $L_{e}=1$, in order to make the analysis tractable mathematically. By using the definition of the channel matrix $\mathbf{H}$ and equations (6) and (7), it is possible to rewrite (46) as:

$$
\begin{gathered}
m_{\text {1-opt }}^{2}\left(\mathbf{w}_{\text {l-opt }}\right)=\left(E_{s}|g|^{2}\right)^{2} \frac{\left|h(0)+h(1)+\ldots+h\left(L_{g}-1\right)\right|^{2}}{C_{y \mid \mathcal{H}_{1}}} \\
=\left(E_{s}|g|^{2}\right)^{2} \frac{\left|\mathbf{1}^{H} \mathbf{H}^{H}\right|^{2}}{C_{y \mid \mathcal{H}_{1}}}
\end{gathered}
$$

where $C_{y \mid \mathcal{H}_{1}}=\mathbf{C}_{\mathbf{y} \mid \mathcal{H}_{1}}$, since, when $M=1$ and $L_{e}=1$, the covariance matrix $\mathbf{C}_{\mathbf{y} \mid \mathcal{H}_{1}}$ becomes a scalar non-negative quantity. Similarly, by exploiting the equations (17), (19) and the inverse of a $(2 \times 2)$ matrix [19], after some algebraic manipulations, (45) can be rewritten as:

$$
\begin{gathered}
m_{\text {wl-opt }}^{2}\left(\mathbf{f}_{\text {wl-opt }}\right)=2\left(E_{s}|g|^{2}\right)^{2}\left\{\frac{C_{y \mid \mathcal{H}_{1}}\left|\mathbf{1}^{H} \mathbf{H}^{H}\right|^{2}}{C_{y \mid \mathcal{H}_{1}}^{2}-\left|C_{y^{*} \mid \mathcal{H}_{1}}\right|^{2}}+\right. \\
\left.-\frac{\operatorname{Re}\left[C_{y^{*} \mid \mathcal{H}_{1}}\left(\mathbf{1}^{H} \mathbf{H}^{H}\right)^{2}\right]}{C_{y \mid \mathcal{H}_{1}}^{2}-\left|C_{y^{*} \mid \mathcal{H}_{1}}\right|^{2}}\right\}
\end{gathered}
$$

We note that $C_{y \mid \mathcal{H}_{1}}^{2}=\left|C_{y \mid \mathcal{H}_{1}}\right|^{2}>\left|C_{y^{*} \mid \mathcal{H}_{1}}\right|^{2}$ as a direct consequence of their definitions (8) and (12), and that the scalar quantity $\operatorname{Re}\left[C_{y^{*} \mid \mathcal{H}_{1}}\left(\mathbf{1}^{H} \mathbf{H}^{H}\right)^{2}\right]$ can assume either positive values or negative values. Therefore, to complete the proof we distinguish between the case in which $\operatorname{Re}\left[C_{y^{*} \mid \mathcal{H}_{1}}\left(\mathbf{1}^{H} \mathbf{H}^{H}\right)^{2}\right]$ is positive and the case in which $\operatorname{Re}\left[C_{y^{*} \mid \mathcal{H}_{1}}\left(\mathbf{1}^{H} \mathbf{H}^{H}\right)^{2}\right]$ is negative.

Case 1. If $\operatorname{Re}\left[C_{y^{*} \mid \mathcal{H}_{1}}\left(\mathbf{1}^{H} \mathbf{H}^{H}\right)^{2}\right]$ assumes positive values, then equation (65), reported at the top of the next page, is obtained by using the notable relation valid for complex number, i.e., $|\operatorname{Re}[x]| \leq|x|$, and the equality $\left|\left(\mathbf{1}^{H} \mathbf{H}^{H}\right)^{2}\right|=\left|\mathbf{1}^{H} \mathbf{H}^{H}\right|^{2}$. Since $C_{y \mid \mathcal{H}_{1}}>\left|C_{y^{*} \mid \mathcal{H}_{1}}\right|$, we can conclude from (65) that:

$$
\begin{aligned}
& m_{\text {wl-opt }}^{2}\left(\mathbf{f}_{\text {wl-opt }}\right) \geq \frac{2\left|\mathbf{1}^{H} \mathbf{H}^{H}\right|^{2}\left(E_{s}|g|^{2}\right)^{2}}{C_{y \mid \mathcal{H}_{1}}+\left|C_{y^{*} \mid \mathcal{H}_{1}}\right|} \\
& >\frac{2\left|\mathbf{1}^{H} \mathbf{H}^{H}\right|^{2}\left(E_{s}|g|^{2}\right)^{2}}{2 C_{y \mid \mathcal{H}_{1}}}=m_{\text {l-opt }}^{2}\left(\mathbf{w}_{\text {l-opt }}\right)
\end{aligned}
$$

Case 2. If $\operatorname{Re}\left[C_{y^{*} \mid \mathcal{H}_{1}}\left(\mathbf{1}^{H} \mathbf{H}^{H}\right)^{2}\right]$ assumes negative values, we can rewrite (64) as:

$$
\begin{aligned}
m_{\text {wl-opt }}^{2}\left(\mathbf{f}_{\text {wl-opt }}\right)= & 2\left(E_{s}|g|^{2}\right)^{2}\left\{\frac{C_{y \mid \mathcal{H}_{1}}\left|\mathbf{1}^{H} \mathbf{H}^{H}\right|^{2}}{C_{y \mid \mathcal{H}_{1}}^{2}-\left|C_{y^{*} \mid \mathcal{H}_{1}}\right|^{2}}+\right. \\
& \left.+\frac{\left|\operatorname{Re}\left[C_{y^{*} \mid \mathcal{H}_{1}}\left(\mathbf{1}^{H} \mathbf{H}^{H}\right)^{2}\right]\right|}{C_{y \mid \mathcal{H}_{1}}^{2}-\left|C_{y^{*} \mid \mathcal{H}_{1}}\right|^{2}}\right\}
\end{aligned}
$$



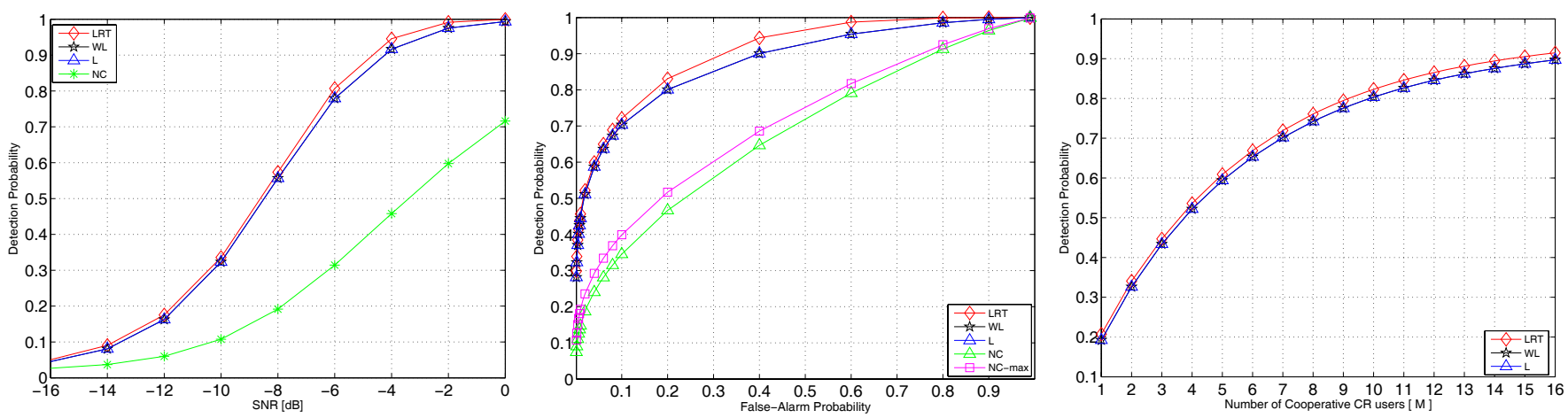

Fig. 9. $P_{d}$ vs SNR, for $P_{f}=10^{-2}$ and $M=4$. Fig. 10. $\quad P_{d}$ vs $P_{f}$, for $M=4$.

Fig. 11. $P_{d}$ vs $M$, for $P_{f}=10^{-2}$.

$$
\begin{aligned}
m_{\text {wl-opt }}^{2}\left(\mathbf{f}_{\text {wl-opt }}\right) & \geq \frac{2\left(E_{s}|g|^{2}\right)^{2}\left\{C_{y \mid \mathcal{H}_{1}}\left|\mathbf{1}^{H} \mathbf{H}^{H}\right|^{2}-\left|C_{y^{*} \mid \mathcal{H}_{1}}\left(\mathbf{1}^{H} \mathbf{H}^{H}\right)^{2}\right|\right\}}{C_{y \mid \mathcal{H}_{1}}^{2}-\left|C_{y^{*} \mid \mathcal{H}_{1}}\right|^{2}}=\frac{2\left|\mathbf{1}^{H} \mathbf{H}^{H}\right|^{2}\left(E_{s}|g|^{2}\right)^{2}\left(C_{y \mid \mathcal{H}_{1}}-\left|C_{y^{*} \mid \mathcal{H}_{1}}\right|\right)}{\left(C_{y \mid \mathcal{H}_{1}}-\left|C_{y^{*} \mid \mathcal{H}_{1}}\right|\right)\left(C_{y \mid \mathcal{H}_{1}}+\left|C_{y^{*} \mid \mathcal{H}_{1}}\right|\right)} \\
& =\frac{2\left|\mathbf{1}^{H} \mathbf{H}^{H}\right|^{2}\left(E_{s}|g|^{2}\right)^{2}}{C_{y \mid \mathcal{H}_{1}}+\left|C_{y^{*} \mid \mathcal{H}_{1}}\right|}
\end{aligned}
$$

The numerator in (67) is greater than
$\left(E_{s}|g|^{2}\right)^{2}\left(2 C_{y \mid \mathcal{H}_{1}}\left|\mathbf{1}^{H} \mathbf{H}^{H}\right|^{2}-2\left|\operatorname{Re}\left[C_{y^{*} \mid \mathcal{H}_{1}}\left(\mathbf{1}^{H} \mathbf{H}^{H}\right)^{2}\right]\right|\right)$ :

$$
\begin{aligned}
& m_{\text {wl-opt }}^{2}\left(\mathbf{f}_{\text {wl-opt }}\right)> \\
& 2\left(E_{s}|g|^{2}\right)^{2} \frac{C_{y \mid \mathcal{H}_{1}}\left|\mathbf{1}^{H} \mathbf{H}^{H}\right|^{2}-\left|\operatorname{Re}\left[C_{y^{*} \mid \mathcal{H}_{1}}\left(\mathbf{1}^{H} \mathbf{H}^{H}\right)^{2}\right]\right|}{C_{y \mid \mathcal{H}_{1}}^{2}-\left|C_{y^{*} \mid \mathcal{H}_{1}}\right|^{2}}
\end{aligned}
$$

By using the relation $|\operatorname{Re}[x]| \leq|x|$, it results $\left|\operatorname{Re}\left[C_{y^{*} \mid \mathcal{H}_{1}}\left(\mathbf{1}^{H} \mathbf{H}^{H}\right)^{2}\right]\right| \leq\left|C_{y^{*} \mid \mathcal{H}_{1}}\right|\left|\mathbf{1}^{H} \mathbf{H}^{H}\right|^{2}$, hence we can proceed following the same reasoning described for Case 1.

\section{APPENDIX B}

\section{ProOF OF PROPOSITION 2}

By exploiting (56), (57) and equation (55) of $\mathbf{f}_{\mathrm{wl}-\mathrm{opt}}=$ $\left[\begin{array}{ll}\mathbf{f}_{1, \mathrm{wl}-\mathrm{opt}}^{T} & \mathbf{f}_{1, \mathrm{wl}-\mathrm{opt}}^{H}\end{array}\right]^{T}$, and by indicating with $\mathbf{C}_{\mathbf{y}_{a} \mid \mathcal{H}_{1}}^{-1} \triangleq$ $\left[\begin{array}{ll}\mathbf{A}_{11} & \mathbf{A}_{12} \\ \mathbf{A}_{21} & \mathbf{A}_{22}\end{array}\right]$, the WL-subvector $\mathbf{f}_{1, \text { wl-opt }}$ can be expressed as:

$$
\mathbf{f}_{1, \mathrm{wl} \text {-opt }}=\frac{\left[\mathbf{A}_{11} \mathbf{H}+\mathbf{A}_{12} \mathbf{H}^{*}\right]\left(\boldsymbol{\eta}_{\mathcal{H}_{1}}-\boldsymbol{\eta}_{\mathcal{H}_{0}}\right)}{\sqrt{2}\left\|\left[\mathbf{A}_{11} \mathbf{H}+\mathbf{A}_{12} \mathbf{H}^{*}\right]\left(\boldsymbol{\eta}_{\mathcal{H}_{1}}-\boldsymbol{\eta}_{\mathcal{H}_{0}}\right)\right\|_{2}^{2}}
$$

By accounting for the inverse of a partitioned matrix [19], $\mathbf{A}_{11}$ and $\mathbf{A}_{12}$ can be expressed as:

$$
\begin{aligned}
& \mathbf{A}_{11}=\left[\mathbf{C}_{\mathbf{y} \mid \mathcal{H}_{1}}-\mathbf{C}_{\mathbf{y}^{*} \mid \mathcal{H}_{1}}\left(\mathbf{C}_{\mathbf{y} \mid \mathcal{H}_{1}}^{*}\right)^{-1} \mathbf{C}_{\mathbf{y}^{*} \mid \mathcal{H}_{1}}^{*}\right]^{-1} \\
& \mathbf{A}_{12}=-\mathbf{C}_{\mathbf{y} \mid \mathcal{H}_{1}}^{-1} \mathbf{C}_{\mathbf{y}^{*} \mid \mathcal{H}_{1}} \mathbf{A}_{11}
\end{aligned}
$$

where the equality for $\mathbf{A}_{12}$ accounts that the diagonal matrix $\mathbf{A}_{11}$ is real. By substituting (70) in (69), and by exploiting the diagonal-nature of the involved matrices, one has:

$$
\mathbf{f}_{1, \mathrm{wl}-\mathrm{opt}}=\frac{\mathbf{A}_{11}\left[\mathbf{H}-\mathbf{C}_{\mathbf{y} \mid \mathcal{H}_{1}}^{-1} \mathbf{C}_{\mathbf{y}^{*} \mid \mathcal{H}_{1}} \mathbf{H}^{*}\right]\left(\boldsymbol{\eta}_{\mathcal{H}_{1}}-\boldsymbol{\eta}_{\mathcal{H}_{0}}\right)}{\sqrt{2} \|\left.\mathbf{A}_{11}\left[\mathbf{H}-\mathbf{C}_{\mathbf{y} \mid \mathcal{H}_{1}}^{-1} \mathbf{C}_{\mathbf{y}^{*} \mid \mathcal{H}_{1}} \mathbf{H}^{*}\right]\left(\boldsymbol{\eta}_{\mathcal{H}_{1}}-\boldsymbol{\eta}_{\mathcal{H}_{0}}\right)\right|_{2} ^{2}}
$$

Moreover, from (53), $\mathbf{C}_{\mathbf{y}^{*} \mid \mathcal{H}_{1}}$ can be rewritten as

$$
\begin{aligned}
\mathbf{C}_{\mathbf{y}^{*} \mid \mathcal{H}_{1}} & =\left[\mathbf{C}_{\mathbf{y} \mid \mathcal{H}_{1}}-\mathbf{R}_{\mathbf{n}}\right]\left(\mathbf{H}^{H}\right)^{-1} \mathbf{H}^{T}= \\
& =\left[\mathbf{C}_{\mathbf{y} \mid \mathcal{H}_{1}}-\mathbf{R}_{\mathbf{n}}\right]\left(\mathbf{H}^{*}\right)^{-1} \mathbf{H}
\end{aligned}
$$

By substituting (72) in (70), and by using the equality $\mathbf{R}_{\mathbf{n}}=$ $\left(\mathbf{C}_{\mathbf{y} \mid \mathcal{H}_{1}}-\mathbf{H ~ C}_{\mathbf{u} \mid \mathcal{H}_{1}} \mathbf{H}^{H}\right)$ one has:

$$
\begin{aligned}
\mathbf{A}_{11} & =\left[\mathbf{R}_{\mathbf{n}}\left(2 \mathbf{I}-\mathbf{C}_{\mathbf{y} \mid \mathcal{H}_{1}}^{-1} \mathbf{R}_{\mathbf{n}}\right)\right]^{-1}= \\
& =\left[\mathbf{I}+\mathbf{C}_{\mathbf{y} \mid \mathcal{H}_{1}}^{-1} \mathbf{C}_{\mathbf{y}^{*} \mid \mathcal{H}_{1}} \mathbf{H}^{-1} \mathbf{H}^{H}\right]^{-1} \mathbf{R}_{\mathbf{n}}^{-1}
\end{aligned}
$$

In addition, by using (72), after some algebraic manipulations, we obtain $\left[\mathbf{H}-\mathbf{C}_{\mathbf{y} \mid \mathcal{H}_{1}}^{-1} \mathbf{C}_{\mathbf{y}^{*} \mid \mathcal{H}_{1}} \mathbf{H}^{*}\right]=\mathbf{C}_{\mathbf{y} \mid \mathcal{H}_{1}}^{-1} \mathbf{R}_{\mathbf{n}} \mathbf{H}=$ $\mathbf{R}_{\mathbf{n}} \mathbf{C}_{\mathbf{y} \mid \mathcal{H}_{1}}^{-1} \mathbf{H}$. By substituting this equality and (73) in (71), $\mathbf{f}_{1, \mathrm{wl}-\mathrm{opt}}$ becomes:

$\mathbf{f}_{1, \mathrm{wl}-\mathrm{opt}}=$

$\frac{\left[\mathbf{I}+\mathbf{C}_{\mathbf{y} \mid \mathcal{H}_{1}}^{-1} \mathbf{C}_{\mathbf{y}^{*} \mid \mathcal{H}_{1}} \mathbf{H}^{-1} \mathbf{H}^{H}\right]^{-1} \mathbf{C}_{\mathbf{y} \mid \mathcal{H}_{1}}^{-1} \mathbf{H}\left(\boldsymbol{\eta}_{\mathcal{H}_{1}}-\boldsymbol{\eta}_{\mathcal{H}_{0}}\right)}{\sqrt{2} \|\left.\left[\mathbf{I}+\mathbf{C}_{\mathbf{y} \mid \mathcal{H}_{1}}^{-1} \mathbf{C}_{\mathbf{y}^{*} \mid \mathcal{H}_{1}} \mathbf{H}^{-1} \mathbf{H}^{H}\right]^{-1} \mathbf{C}_{\mathbf{y} \mid \mathcal{H}_{1}}^{-1} \mathbf{H}\left(\boldsymbol{\eta}_{\mathcal{H}_{1}}-\boldsymbol{\eta}_{\mathcal{H}_{0}}\right)\right|_{2} ^{2}}$

By accounting for (54) and (49), the following equality holds:

$$
\mathbf{C}_{\mathbf{y} \mid \mathcal{H}_{1}}^{-1} \mathbf{H}\left(\boldsymbol{\eta}_{\mathcal{H}_{1}}-\boldsymbol{\eta}_{\mathcal{H}_{0}}\right)=\|\left.\mathbf{C}_{\mathbf{y} \mid \mathcal{H}_{1}}^{-1} \mathbf{H}\left(\boldsymbol{\eta}_{\mathcal{H}_{1}}-\boldsymbol{\eta}_{\mathcal{H}_{0}}\right)\right|_{2} ^{2} \mathbf{w}_{\text {l-opt }}
$$

For (75), the denominator of (74) can be written as: $\quad \sqrt{2}\left\|\mathbf{C}_{\mathbf{y} \mid \mathcal{H}_{1}}^{-1} \mathbf{H}\left(\boldsymbol{\eta}_{\mathcal{H}_{1}}-\boldsymbol{\eta}_{\mathcal{H}_{0}}\right)\right\|_{2}^{2} \|[\mathbf{I} \quad+$ $\left.\mathbf{C}_{\mathbf{y} \mid \mathcal{H}_{1}}^{-1} \mathbf{C}_{\mathbf{y}^{*} \mid \mathcal{H}_{1}} \mathbf{H}^{-1} \mathbf{H}^{H}\right]^{-1} \mathbf{w}_{\text {l-opt }} \|_{2}^{2}$. By using this equality and (75) in (74), the proof is given:

$\mathbf{f}_{1, \mathrm{wl} \text {-opt }}=\frac{\left[\mathbf{I}+\mathbf{C}_{\mathbf{y} \mid \mathcal{H}_{1}}^{-1} \mathbf{C}_{\mathbf{y}^{*} \mid \mathcal{H}_{1}} \mathbf{H}^{-1} \mathbf{H}^{H}\right]^{-1} \mathbf{w}_{\text {l-opt }}}{\sqrt{2}\left\|\left[\mathbf{I}+\mathbf{C}_{\mathbf{y} \mid \mathcal{H}_{1}}^{-1} \mathbf{C}_{\mathbf{y}^{*} \mid \mathcal{H}_{1}} \mathbf{H}^{-1} \mathbf{H}^{H}\right]^{-1} \mathbf{w}_{\text {l-opt }}\right\|_{2}^{2}}$

\section{REFERENCES}

[1] S. Haykin, "Cognitive radio: brain-empowered wireless communications," IEEE J. Sel. Areas Commun., vol. 23, no. 2, pp. 201-220, Feb. 2005.

[2] A. Ghasemi and E. Sousa, "Collaborative spectrum sensing for opportunistic access in fading environments," in Proc. IEEE DySPAN, Nov. 2005, pp. 131-136. 
[3] K. Ben Letaief and W. Zhang, "Cooperative communications for cognitive radio networks," Proc. IEEE, vol. 97, no. 5, pp. 878-893, May 2009.

[4] J. Ma, G. Li, and B. H. Juang, "Signal processing in cognitive radio," Proc. IEEE, vol. 97, no. 5, pp. 805-823, May 2009.

[5] J. Man and Y. Li, "Soft combination and detection for cooperative spectrum sensing in cognitive radio natworks," in Proc. IEEE GLOBECOM, Nov. 2007, pp. 3139-3143.

[6] Z. Quan, S. Cui, and A. H. Sayed, "An optimal strategy for cooperative spectrum sensing in cognitive radio networks," in Proc. IEEE GLOBECOM, Nov. 2007, pp. 2947-2951.

[7] J. Ma, G. Zhao, and Y. Li, "Soft combination and detection for cooperative spectrum sensing in cognitive radio networks," IEEE Trans. Wireless Commun., vol. 7, no. 11, pp. 4502-4507, Nov. 2008.

[8] Z. Quan, S. Cui, and A. H. Sayed, "Optimal linear cooperation for spectrum sensing in cognitive radio networks," IEEE J. Sel. Topics Signal Process., vol. 2, no. 1, pp. 28-40, Feb. 2008.

[9] W. Zhang and K. Letaief, "Cooperative spectrum sensing with transmit and relay diversity in cognitive radio networks," IEEE Trans. Wireless Commun., vol. 7, no. 12, pp. 4761-4766, Dec. 2008.

[10] W. Zhang, R. Mallik, and K. Letaief, "Optimization of cooperative spectrum sensing with energy detection in cognitive radio networks," IEEE Trans. Wireless Commun., vol. 8, no. 12, pp. 5761-5766, Dec. 2009.

[11] M. Renzo, L. Imbriglio, F. Graziosi, and F. Santucci, "Distributed data fusion over correlated log-normal sensing and reporting channels: application to cognitive radio networks," IEEE Trans. Wireless Commun., vol. 8, no. 12, pp. 5813-5821, Dec. 2009.

[12] B. Kasiri and J. Cai, "Effects of correlated shadowing on soft decision fusion in cooperative spectrum sensing," in Proc. IEEE INFOCOM, Mar. 2010, pp. 1-6.

[13] Y. Zou, Y.-D. Yao, and B. Zheng, "Outage probability analysis of cognitive transmissions: impact of spectrum sensing overhead," IEEE Trans. Wireless Commun., vol. 9, no. 8, pp. 2676-2688, Aug. 2010.

[14] I. F. Akyildiz, B. F. Lo, and R. Balakrishnan, "Cooperative spectrum sensing in cognitive radio networks: a survey," Physical Commun. (Elsevier) J., vol. 4, no. 1, pp. 40-62, Mar. 2011.

[15] S. M. Kay, Fundamentals of Statistical Signal Processing II: Detection Theory. Prentice-Hall, 1998.

[16] P. Schreier and L. Scharf, "Second-order analysis of improper complex random vectors and processes," IEEE Trans. Signal Process., vol. 51, no. 3, pp. 714-725, Mar. 2003.

[17] B. Picinbono and P. Chevalier, "Widely linear estimation with complex data," IEEE Trans. Signal Process., vol. 43, no. 8, pp. 2030-2033, Aug. 1995.

[18] Y.-C. Liang, Y. Zeng, E. C. Peh, and A. T. Hoang, "Sensing-throughput tradeoff for cognitive radio networks," IEEE Trans. Wireless Commun., vol. 7, no. 4, pp. 1326-1337, Apr. 2008.

[19] R. A. Horn and C. R. Johnson, Matrix Analysis. Cambridge University Press, 1990.

[20] A. S. Cacciapuoti, G. Gelli, L. Paura, and F. Verde, "Finite-sample performance analysis of widely-linear multiuser receivers for DS-CDMA systems," IEEE Trans. Signal Process., vol. 56, no. 4, pp. 1572-1588, Apr. 2008.

[21] B. Picinbono, "On deflection as a performance criterion in detection," IEEE Trans. Aerosp. Electron. Syst., vol. 31, no. 3, pp. 1072-1081, July 1995.

[22] S. J. Shellhammer, S. S. N, R. Tandra, and J. Tomcik, "Performance of power detector sensors of DTV signals in IEEE 802.22 WRANs," in Proc. TAPAS, 2006.

[23] H. Kim and K. Shin, "In-band spectrum sensing in IEEE 802.22 WRANs for incumbent protection," IEEE Trans. Mobile Computing, vol. 9, no. 12, pp. 1766-1779, Dec. 2010.

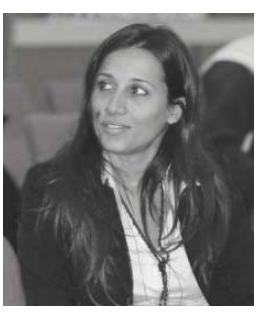

Angela Sara Cacciapuoti received the Dr. Eng. degree summa cum laude in Telecommunications Engineering in 2005, and the Ph.D degree with score "excellent" in Electronic and Telecommunications Engineering in 2009, both from University of Naples Federico II. Since 2008, she is with the Dept. of Biomedical, Electronic and Telecommunications Engineering (DIBET), University of Naples Federico II, as postdoctoral research fellow. From 2010 to 2011, she has been with Broadband Wireless Networking Lab, Georgia Institute of Technology, as visiting researcher. In 2011, she has also been with NaNoNetworking Center in Catalunya (N3Cat), Universitat Politecnica de Catalunya, as visiting research. Her research activities lie in the area of statistical signal processing, digital communications, and communication systems. In particular, her current interests are focused on non-stationary signal processing, cognitive networks and wireless communications cross- layering.

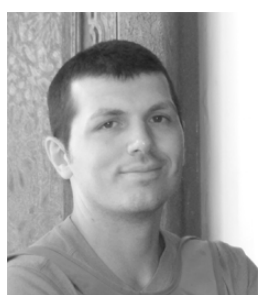

Marcello Caleffi received the Dr. Eng. degree summa cum laude in Computer Science Engineering from University of Lecce in 2005, and the Ph.D. degree in Electronic and Telecommunications Engineering from University of Naples Federico II in 2009. Since 2008, he is with the Dept. of Biomedical, Electronic and Telecommunications Engineering, University of Naples Federico II, as postdoctoral research fellow. From 2010 to 2011, he was with Broadband Wireless Networking Lab, Georgia Institute of Technology, as visiting researcher. In 2011, he was with the NaNoNetworking Center in Catalunya (N3Cat), Universitat Politecnica de Catalunya, as visiting researcher. His research activities lie in the area of wireless networking. In particular, his current interests are focused on cognitive ad hoc networks and human-enabled wireless networks.

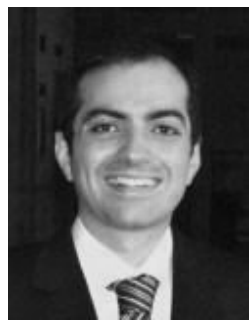

Domenico Izzo received the Dr. Eng. degree summa cum laude in Telecommunication Engineering in 2009 from University of Naples Federico II. From 2010 to 2011, he was a scholarship holder at Consorzio Nazionale Interuniversitario per le Telecomunicazioni (CNIT), cooperating with the Dep. of Biomedical, Electronic and Telecommunications Engineering, University of Naples Federico II, on topics related to cognitive radio networks.

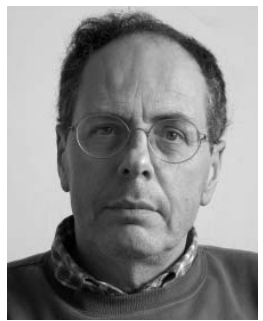

Luigi Paura received the Dr. Eng. degree summa cum laude in Electronic Engineering in 1974 from University of Napoli Federico II. From 1979 to 1984, he was with the Dept. of Biomedical, Electronic and Telecommunication Engineering, University of Napoli Federico II, first as an Assistant Professor and then as an Associate Professor. Since 1994, he has been a Full Professor of Telecommunications: first with the Dept. of Mathematics, University of Lecce, Italy; then with the Dept. of Information Engineering, Second University of Naples; and finally, from 1998 he has been with the Dept. of Biomedical, Electronic and Telecommunication Engineering, University of Naples Federico II. $\mathrm{He}$ also held teaching positions at University of Salerno, at University of Sannio, and at University Parthenope of Naples. In 1985-86 and 1991 he was a Visiting Researcher at Signal and Image Processing Laboratory, University of California, Davis. At the present time, his research activities are mainly concerned with statistical signal processing, digital communication systems, and cognitive networks. 